\title{
Regularity of plurisubharmonic upper envelopes in big cohomology classes
}

\author{
Robert Berman \\ Department of Mathematics, \\ Chalmers University of Technology, \\ Eklandag. 86, \\ SE-412 96 Göteborg, Sweden \\ e-mail: robertb@chalmers.se
}

\author{
Jean-Pierre Demailly \\ Université de Grenoble I, \\ Département de Mathématiques, \\ Institut Fourier, BP 74, \\ 38402 Saint-Martin d'Hères, France \\ demailly@fourier.ujf-grenoble.fr
}

\begin{abstract}
The goal of this work is to prove the regularity of certain quasiplurisubharmonic upper envelopes. Such envelopes appear in a natural way in the construction of hermitian metrics with minimal singularities on a big line bundle over a compact complex manifold. We prove that the complex Hessian forms of these envelopes are locally bounded outside an analytic set of singularities. It is furthermore shown that a parametrized version of this result yields a priori inequalities for the solution of the Dirichlet problem for a degenerate MongeAmpère operator; applications to geodesics in the space of Kähler metrics are discussed. A similar technique provides a logarithmic modulus of continuity for Tsuji's "supercanonical" metrics, which generalize a well-known construction of Narasimhan-Simha.
\end{abstract}

Résumé. Le but de ce travail est de démontrer la régularité de certaines enveloppes supérieures de fonctions quasi-plurisousharmoniques. De telles enveloppes apparaissent naturellement dans la construction des métriques hermitiennes à singularités minimales sur un fibré en droites gros au dessus d'une variété complexe compacte. Nous montrons que ces enveloppes possèdent un Hessien complexe localement borné en dehors d'un ensemble analytique de singularités; par ailleurs, une version avec paramètres de ce résultat permet d'obtenir des inégalités a priori pour la solution du problème de Dirichlet relatif à un opérateur de MongeAmpère dégénéré. Une technique similaire fournit un module de continuité logarithmique pour les métriques "super-canoniques" de Tsuji, lesquelles généralisent une construction bien connue de Narasimhan-Simha.

Key words. Plurisubharmonic function, upper envelope, hermitian line bundle, singular metric, logarithmic poles, Legendre-Kiselman transform, pseudo-effective cone, volume, Monge-Ampère measure, supercanonical metric, Ohsawa-Takegoshi theorem.

Mots-clés. Fonction plurisubharmonique, enveloppe supérieure, fibré en droites hermitien, métrique singulière, pôles logarithmiques, transformée de LegendreKiselman, cône pseudo-effectif, volume, mesure de Monge-Ampère, métrique super-canonique, théorème de Ohsawa-Takegoshi.

AMS Classification. 32F07, 32J25, 14B05, 14C30 


\section{Main regularity theorem}

Let $X$ be a compact complex manifold and $\omega$ a hermitian metric on $X$, viewed as a smooth positive $(1,1)$-form. As usual we put $d^{c}=\frac{1}{4 i \pi}(\partial-\bar{\partial})$ so that $d d^{c}=\frac{1}{2 i \pi} \partial \bar{\partial}$. Consider the $d d^{c}$-cohomology class $\{\alpha\}$ of a smooth real $d$-closed form $\alpha$ of type $(1,1)$ on $X$ [in general, one has to consider the BottChern cohomology group for which boundaries are $d d^{c}$-exact $(1,1)$-forms $d d^{c} \varphi$, but in the case $X$ is Kähler, this group is isomorphic to the Dolbeault cohomology group $H^{1,1}(X)$ ]. Recall that a function $\psi$ is said to be quasi-plurisubharmonic (or quasi-psh) if and only $i d d^{c} \psi$ is locally bounded from below, or equivalently, if it can be written locally as a sum $\psi=\varphi+u$ of a psh function $\varphi$ and a smooth function $u$. More precisely, it is said to be $\alpha$-plurisubharmonic (or $\alpha$-psh) if $\alpha+d d^{c} \psi \geqslant 0$. We denote by $\operatorname{PSH}(X, \alpha)$ the set of $\alpha$-psh functions on $X$.

(1.1) Definition. The class $\{\alpha\} \in H^{1,1}(X, \mathbb{R})$ is said to be pseudo-effective if it contains a closed (semi-)positive current $T=\alpha+d d^{c} \psi \geqslant 0$, and big if it contains a closed "Kähler current" $T=\alpha+d d^{c} \psi$ such that $T \geqslant \varepsilon \omega>0$ for some $\varepsilon>0$.

From now on in this section, we assume that $\{\alpha\}$ is big. We know by [Dem92] that we can then find $T_{0} \in\{\alpha\}$ of the form

$$
T_{0}=\alpha+d d^{c} \psi_{0} \geqslant \varepsilon_{0} \omega
$$

with a possibly slightly smaller $\varepsilon_{0}>0$ than the $\varepsilon$ in the definition, and $\psi_{0}$ a quasi-psh function with analytic singularities, i.e. locally

$$
\psi_{0}=c \log \sum\left|g_{j}\right|^{2}+u, \quad \text { where } c>0, u \in C^{\infty}, g_{j} \text { holomorphic. }
$$

By [DP04], $X$ carries such a class $\{\alpha\}$ if and only if $X$ is in the Fujiki class $\mathcal{C}$ of smooth varieties which are bimeromorphic to compact Kähler manifolds. Our main result is

(1.4) Theorem. Let $X$ be a compact complex manifold in the Fujiki class $\mathrm{C}$, and let $\alpha$ be a smooth closed form of type $(1,1)$ on $X$ such that the cohomology class $\{\alpha\}$ is big. Pick $T_{0}=\alpha+d d^{c} \psi_{0} \in\{\alpha\}$ satisfying (1.2) and (1.3) for some hermitian metric $\omega$ on $X$, and let $Z_{0}$ be the analytic set $Z_{0}=\psi_{0}^{-1}(-\infty)$. Then the upper envelope

$$
\varphi:=\sup \{\psi \leqslant 0, \psi \alpha-p s h\}
$$

is a quasi-plurisubharmonic function which has locally bounded second order derivatives $\partial^{2} \varphi / \partial z_{j} \partial \bar{z}_{k}$ on $X \backslash Z_{0}$, and moreover, for suitable constants $C, B>0$, there is a global bound

$$
\left|d d^{c} \varphi\right|_{\omega} \leqslant C\left(\left|\psi_{0}\right|+1\right)^{2} e^{B\left|\psi_{0}\right|}
$$

which explains how these derivatives blow up near $Z_{0}$. In particular $\varphi$ is $C^{1,1-\delta}$ on $X \backslash Z_{0}$ for every $\delta>0$, and the second derivatives $D^{2} \varphi$ are in $L_{\mathrm{loc}}^{p}\left(X \backslash Z_{0}\right)$ for every $p>0$. 
An important special case is the situation where we have a hermitian line bundle $\left(L, h_{L}\right)$ and $\alpha=\Theta_{L, h_{L}}$, with the assumption that $L$ is big, i.e. that there exists a singular hermitian $h_{0}=h_{L} e^{-\psi_{0}}$ which has analytic singularities and a curvature current $\Theta_{L, h_{0}}=\alpha+d d^{c} \psi_{0} \geqslant \varepsilon_{0} \omega$. We then infer that the metric with minimal singularities $h_{\text {min }}=h_{L} e^{-\varphi}$ has the regularity properties prescribed by Theorem 1.4 outside of the analytic set $Z_{0}=\psi_{0}^{-1}(-\infty)$. In fact, [Ber07, Theorem 3.4 (a)] proves in this case the slightly stronger result that $\varphi$ in $C^{1,1}$ on $X \backslash Z_{0}$ (using the fact that $X$ is then Moishezon and that the total space of $L^{*}$ has a lot of holomorphic vector fields). The present approach is by necessity different, since we can no longer rely on the existence of vector fields when $X$ is not algebraic. Even then, our proof will be in fact somewhat simpler.

Proof. Notice that in order to get a quasi-psh function $\varphi$ we should a priori replace $\varphi$ by its upper semi-continuous regularization $\varphi^{*}(z)=\lim \sup _{\zeta \rightarrow z} \varphi(\zeta)$, but since $\varphi^{*} \leqslant 0$ and $\varphi^{*}$ is $\alpha$-psh as well, $\psi=\varphi^{*}$ contributes to the envelope and therefore $\varphi=\varphi^{*}$. Without loss of generality, after substracting a constant to $\psi_{0}$, we may assume $\psi_{0} \leqslant 0$. Then $\psi_{0}$ contributes to the upper envelope and therefore $\varphi \geqslant \psi_{0}$. This already implies that $\varphi$ is locally bounded on $X \backslash Z_{0}$. Following [Dem94], for every $\delta>0$, we consider the regularization operator

$$
\psi \mapsto \rho_{\delta} \psi
$$

defined by $\rho_{\delta} \psi(z)=\Psi(z, \delta)$ and

$$
\Psi(z, w)=\int_{\zeta \in T_{X, z}} \psi\left(\operatorname{exph}_{z}(w \zeta)\right) \chi\left(|\zeta|^{2}\right) d V_{\omega}(\zeta), \quad(z, w) \in X \times \mathbb{C}
$$

where exph $: T_{X} \rightarrow X, T_{X, z} \ni \zeta \mapsto \operatorname{exph}_{z}(\zeta)$ is the formal holomorphic part of the Taylor expansion of the exponential map of the Chern connection on $T_{X}$ associated with the metric $\omega$, and $\chi: \mathbb{R} \rightarrow \mathbb{R}_{+}$is a smooth function with support in ] $-\infty, 1]$ defined by

$$
\chi(t)=\frac{C}{(1-t)^{2}} \exp \frac{1}{t-1} \quad \text { for } t<1, \quad \chi(t)=0 \quad \text { for } t \geqslant 1
$$

with $C>0$ adjusted so that $\int_{|x| \leqslant 1} \chi\left(|x|^{2}\right) d x=1$ with respect to the Lebesgue measure $d x$ on $\mathbb{C}^{n}$. Also, $d V_{\omega}(\zeta)$ denotes the standard hermitian Lebesgue measure on $\left(T_{X}, \omega\right)$. Clearly $\Psi(z, w)$ depends only on $|w|$. With the relevant change of notation, the estimates proved in sections 3 and 4 of [Dem94] (see especially Theorem 4.1 and estimates (4.3), (4.5) therein) show that if one assumes $\alpha+d d^{c} \psi \geqslant 0$, there are constants $\delta_{0}, K>0$ such that for $(z, w) \in X \times \mathbb{C}$

$$
\begin{aligned}
& {\left[0, \delta_{0}\right] \ni t \mapsto \Psi(z, t)+K t^{2} \quad \text { is increasing }} \\
& \alpha(z)+d d^{c} \Psi(z, w) \geqslant-A \lambda(z,|w|)|d z|^{2}-K\left(|w|^{2}|d z|^{2}+|d z||d w|+|d w|^{2}\right)
\end{aligned}
$$


where $A=\sup _{|\zeta| \leqslant 1,|\xi| \leqslant 1}\left\{-c_{j k \ell m} \zeta_{j} \bar{\zeta}_{k} \xi_{\ell} \bar{\xi}_{m}\right\}$ is a bound for the negative part of the curvature tensor $\left(c_{j k \ell m}\right)$ of $\left(T_{X}, \omega\right)$ and

$$
\lambda(z, t)=\frac{d}{d \log t}\left(\Psi(z, t)+K t^{2}\right) \underset{t \rightarrow 0_{+}}{\longrightarrow} \nu(\psi, z) \quad \text { (Lelong number) }
$$

In fact, this is clear from [Dem94] if $\alpha=0$, and otherwise we simply apply the above estimates (1.7-1.9) locally to $u+\psi$ where $u$ is a local potential of $\alpha$ and then subtract the resulting regularization $U(z, w)$ of $u$ which is such that

$$
d d^{c}(U(z, w)-u(z))=O\left(|w|^{2}|d z|^{2}+|w||d z||d w|+|d w|^{2}\right)
$$

because the left hand side is smooth and $U(z, w)-u(z)=O\left(|w|^{2}\right)$. As a consequence, the regularization operator $\rho_{\delta}$ transforms quasi-psh functions into quasi-psh functions, while providing very good control on the complex Hessian. We exploit this, again quite similarly as in [Dem94], by introducing the KiselmanLegendre transform (cf. [Kis78, Kis94])

$$
\left.\left.\psi_{c, \delta}(z)=\inf _{t \in] 0, \delta]} \rho_{t} \psi(z)+K t^{2}-K \delta^{2}-c \log \frac{t}{\delta}, \quad c>0, \delta \in\right] 0, \delta_{0}\right]
$$

We need the following basic lower bound on the Hessian form.

(1.12) Lemma. For all $c>0$ and $\left.\delta \in] 0, \delta_{0}\right]$ we have

$$
\alpha+d d^{c} \psi_{c, \delta} \geqslant-\left(A \min (c, \lambda(z, \delta))+K \delta^{2}\right) \omega
$$

Proof of lemma. In general an infimum $\inf _{\eta \in E} u(z, \eta)$ of psh functions $z \mapsto u(z, \eta)$ is not psh, but this is the case if $u(z, \eta)$ is psh with respect to $(z, \eta)$ and $u(z, \eta)$ depends only on Re $\eta$ - in which case it is actually a convex function of $\operatorname{Re} \eta$ - this fundamental fact is known as Kiselman's infimum principle. We apply it here by putting $w=e^{\eta}$ and $t=|w|=e^{\operatorname{Re} \eta}$. At all points of $E_{c}(\psi)=\{z \in X ; \nu(\psi, z) \geqslant c\}$ the infinimum occurring in (1.11) is attained at $t=0$. However, for $z \in X \backslash E_{c}(\psi)$ it is attained for $t=t_{\min }$ where

$$
\begin{cases}t_{\min }=\delta & \text { if } \lambda(z, \delta) \leqslant c \\ t_{\min }<\delta & \text { such that } c=\lambda\left(z, t_{\min }\right)=\frac{d}{d t}\left(\Psi(z, t)+K t^{2}\right)_{t=t_{\min }} \text { if } \lambda(z, \delta)>c .\end{cases}
$$

In a neighborhood of such a point $z \in X \backslash E_{c}(\psi)$, the infimum coincides with the infimum taken for $t$ close to $t_{\min }$, and all functions involved have (modulo addition of $\alpha)$ a Hessian form bounded below by $-\left(A \lambda\left(z, t_{\min }\right)+K \delta^{2}\right) \omega$ by (1.8). Since $\lambda\left(z, t_{\min }\right) \leqslant \min (c, \lambda(z, \delta))$, we get the desired estimate on the dense open set $X \backslash E_{c}(\psi)$ by Kiselman's infimum principle. However $\psi_{c, \delta}$ is quasi-psh on $X$ and $E_{c}(\psi)$ is of measure zero, so the estimate is in fact valid on all of $X$, in the sense of currents. 
We now proceed to complete the proof or Theorem 1.4. Lemma 1.12 implies the more brutal estimate

$$
\left.\left.\alpha+d d^{c} \psi_{c, \delta} \geqslant-\left(A c+K \delta^{2}\right) \omega \quad \text { for } \delta \in\right] 0, \delta_{0}\right]
$$

Consider the convex linear combination

$$
\theta=\frac{A c+K \delta^{2}}{\varepsilon_{0}} \psi_{0}+\left(1-\frac{A c+K \delta^{2}}{\varepsilon_{0}}\right) \varphi_{c, \delta}
$$

where $\varphi$ is the upper envelope of all $\alpha$-psh functions $\psi \leqslant 0$. Since $\alpha+d d^{c} \varphi \geqslant 0$, (1.2) and (1.13) imply

$$
\alpha+d d^{c} \theta \geqslant\left(A c+K \delta^{2}\right) \omega-\left(1-\frac{A c+K \delta^{2}}{\varepsilon_{0}}\right)\left(A c+K \delta^{2}\right) \omega \geqslant 0 .
$$

Also $\varphi \leqslant 0$ and therefore $\varphi_{c, \delta} \leqslant \rho_{\delta} \varphi \leqslant 0$ and $\theta \leqslant 0$ likewise. In particular $\theta$ contributes to the envelope and as a consequence we get $\varphi \geqslant \theta$. Coming back to the definition of $\varphi_{c, \delta}$, we infer that for every point $z \in X \backslash Z_{0}$ and every $\delta>0$, there exists $t \in] 0, \delta]$ such that

$$
\begin{aligned}
\varphi(z) & \geqslant \frac{A c+K \delta^{2}}{\varepsilon_{0}} \psi_{0}(z)+\left(1-\frac{A c+K \delta^{2}}{\varepsilon_{0}}\right)\left(\rho_{t} \varphi(z)+K t^{2}-K \delta^{2}-c \log t / \delta\right) \\
& \geqslant \frac{A c+K \delta^{2}}{\varepsilon_{0}} \psi_{0}(z)+\left(\rho_{t} \varphi(z)+K t^{2}-K \delta^{2}-c \log t / \delta\right)
\end{aligned}
$$

(using the fact that the infimum is $\leqslant 0$ and reached for some $t \in] 0, \delta]$, as $t \mapsto \rho_{t} \varphi(z)$ is bounded for $\left.z \in X \backslash Z_{0}\right)$. Therefore we get

$$
\rho_{t} \varphi(z)+K t^{2} \leqslant \varphi(z)+K \delta^{2}-\left(A c+K \delta^{2}\right) \varepsilon_{0}^{-1} \psi_{0}(z)+c \log \frac{t}{\delta} .
$$

Since $t \mapsto \rho_{t} \varphi(z)+K t^{2}$ is increasing and equal to $\varphi(z)$ for $t=0$, we infer that

$$
K \delta^{2}-\left(A c+K \delta^{2}\right) \varepsilon_{0}^{-1} \psi_{0}(z)+c \log \frac{t}{\delta} \geqslant 0,
$$

or equivalently, since $\psi_{0} \leqslant 0$,

$$
t \geqslant \delta \exp \left(-\left(A+K \delta^{2} / c\right) \varepsilon_{0}^{-1}\left|\psi_{0}(z)\right|-K \delta^{2} / c\right) .
$$

Now, (1.14) implies the weaker estimate

$$
\rho_{t} \varphi(z) \leqslant \varphi(z)+K \delta^{2}+\left(A c+K \delta^{2}\right) \varepsilon_{0}^{-1}\left|\psi_{0}(z)\right|,
$$

hence, by combining the last two inequalities, we get

$$
\begin{aligned}
& \frac{\rho_{t} \varphi(z)-\varphi(z)}{t^{2}} \\
& \quad \leqslant K\left(1+\left(\frac{A c}{K \delta^{2}}+1\right) \varepsilon_{0}^{-1}\left|\psi_{0}(z)\right|\right) \exp \left(2\left(A+K \frac{\delta^{2}}{c}\right) \varepsilon_{0}^{-1}\left|\psi_{0}(z)\right|+2 K \frac{\delta^{2}}{c}\right) .
\end{aligned}
$$


We exploit this by letting $0<t \leqslant \delta$ and $c$ tend to 0 , in such a way that $A c / K \delta^{2}$ converges to a positive limit $\ell$ (if $A=0$, just enlarge $A$ slightly and then let $A \rightarrow 0$ ). In this way we get for every $\ell>0$

$$
\begin{aligned}
\liminf _{t \rightarrow 0_{+}} & \frac{\rho_{t} \varphi(z)-\varphi(z)}{t^{2}} \\
& \leqslant K\left(1+(\ell+1) \varepsilon_{0}^{-1}\left|\psi_{0}(z)\right|\right) \exp \left(2 A\left(\left(1+\ell^{-1}\right) \varepsilon_{0}^{-1}\left|\psi_{0}(z)\right|+\ell^{-1}\right)\right) .
\end{aligned}
$$

The special (essentially optimal) choice $\ell=\varepsilon_{0}^{-1}\left|\psi_{0}(z)\right|+1$ yields

$$
\liminf _{t \rightarrow 0_{+}} \frac{\rho_{t} \varphi(z)-\varphi(z)}{t^{2}} \leqslant K\left(\varepsilon_{0}^{-1}\left|\psi_{0}(z)\right|+1\right)^{2} \exp \left(2 A\left(\varepsilon_{0}^{-1}\left|\psi_{0}(z)\right|+1\right)\right) .
$$

Now, putting as usual $\nu(\varphi, z, r)=\frac{1}{\pi^{n-1} r^{2 n-2} /(n-1) !} \int_{B(z, r)} \Delta \varphi(\zeta) d \zeta$, we infer from estimate (4.5) of [Dem94] the Lelong-Jensen like inequality

$$
\begin{aligned}
\rho_{t} \varphi(z)-\varphi(z) & =\int_{0}^{t} \frac{d}{d \tau} \Phi(z, \tau) d \tau \\
& \geqslant \int_{0}^{t} \frac{d \tau}{\tau}\left(\int_{B(0,1)} \nu(\varphi, z, \tau|\zeta|) \chi\left(|\zeta|^{2}\right) d \zeta-O\left(\tau^{2}\right)\right) \\
& \geqslant c(a) \nu(\varphi, z, a t)-C_{2} t^{2} \quad \text { where } a<1, c(a)>0 \text { and } C_{2} \gg 1, \\
& =\frac{c^{\prime}(a)}{t^{2 n-2}} \int_{B(z, a t)} \Delta \varphi(\zeta) d \zeta-C_{2} t^{2}
\end{aligned}
$$

where the third line is obtained by integrating for $\tau \in\left[a^{1 / 2} t, t\right]$ and for $\zeta$ in the corona $a^{1 / 2}<|\zeta|<a^{1 / 4}$ (here we assume that $\chi$ is taken to be decreasing with $\chi(t)>0$ for all $t<1$, and we compute the laplacian $\Delta$ in normalized coordinates at $z$ given by $\left.\zeta \mapsto \operatorname{exph}_{z}(\zeta)\right)$. Hence by Lebesgue's theorem on the existence almost everywhere of the density of a positive measure (see e.g. [Rud66], 7.14), we find

$$
\lim _{t \rightarrow 0_{+}} \frac{1}{t^{2}}\left(\rho_{t} \varphi(z)-\varphi(z)\right) \geqslant c^{\prime \prime}\left(\Delta_{\omega} \varphi\right)_{\mathrm{ac}}(z)-C_{2} \quad \text { a.e. on } X
$$

where the ac subscript means the absolutely continuous part of the measure $\Delta_{\omega} \varphi$. By combining (1.15) and (1.17) and using the quasi-plurisubharmonicity of $\varphi$ we conclude that

$$
\left|d d^{c} \varphi\right|_{\omega} \leqslant \Delta_{\omega} \varphi+C_{3} \leqslant C\left(\left|\psi_{0}\right|+1\right)^{2} e^{2 A \varepsilon_{0}^{-1} \psi_{0}(z)} \quad \text { a.e. on } X \backslash Z_{0}
$$

for some constant $C>0$. There cannot be any singular measure part $\mu$ in $\Delta_{\omega} \varphi$ either, since we now that the Lebesgue density would then be equal to $+\infty \mu$-a.e. ([Rud66], 7.15), in contradiction with (1.15). This gives the required estimates for the complex derivatives $\partial^{2} \varphi / \partial z_{j} \partial \bar{z}_{k}$. The other real derivatives $\partial^{2} \varphi / \partial x_{i} \partial x_{j}$ 
are obtained from $\Delta \varphi=\sum_{k} \partial^{2} \varphi / \partial z_{k} \partial \bar{z}_{k}$ via singular integral operators, and it is well-known that these operate boundedly on $L^{p}$ for all $p<\infty$. Theorem (1.4) follows.

(1.18) Remark. The proof gave us in fact the very explicit value $B=2 A \varepsilon_{0}^{-1}$, where $A$ is an upper bound of the negative part of the curvature of $\left(T_{X}, \omega\right)$. The slightly more refined estimates obtained in [Dem94] show that we could even replace $B$ by the possibly smaller constant $B_{\eta}=2\left(A^{\prime}+\eta\right) \varepsilon_{0}^{-1}$ where

$$
A^{\prime}=\sup _{|\zeta|=1,|\xi|=1, \zeta \perp \xi}-c_{j k \ell m} \zeta_{j} \bar{\zeta}_{k} \xi_{\ell} \bar{\xi}_{m}
$$

and the dependence of the other constants on $\eta$ could then be made explicit.

(1.19) Remark. In Theorem (1.4), one can replace the assumption that $\alpha$ is smooth by the assumption that $\alpha$ has $L^{\infty}$ coefficients. In fact, we used the smoothness of $\alpha$ only as a cheap argument to get the validity of estimate (1.10) for the local potentials $u$ of $\alpha$. However, the results of [Dem94] easily imply the same estimates when $\alpha$ is $L^{\infty}$, as both $u$ and $-u$ are then quasi-psh; this follows e.g. from (1.8) applied with respect to a smooth $\alpha_{\infty}$ and $\psi= \pm u$ if we observe that $\lambda(z,|w|)=O\left(|w|^{2}\right)$ when $\left|d d^{c} \psi\right|_{\omega}$ is bounded. Therefore, only the constant $K$ will be affected in the proof.

\section{Applications to volume and Monge-Ampère measures}

Recall that the volume of a big class $\{\alpha\}$ is defined, in the work [Bou02] of S. Boucksom, as

$$
\operatorname{Vol}(\{\alpha\})=\sup _{T} \int_{X \backslash \operatorname{sing}(T)} T^{n},
$$

with $T$ ranging over all positive currents in the class $\{\alpha\}$ with analytic singularities, whose locus is denoted by $\operatorname{sing}(T)$. If the class is not big then the volume is defined to be zero. With this definition, it is clear that $\{\alpha\}$ is big precisely when $\operatorname{Vol}(\{\alpha\})>0$.

Now fix a smooth representative $\alpha$ in a pseudo-effective class $\{\alpha\}$. We then obtain a uniquely defined $\alpha$-plurisubharmonic function $\varphi=\psi_{\min } \geqslant 0$ with minimal singularities defined as in Theorem (1.4) by

$$
\varphi:=\sup \{\psi \leqslant 0, \psi \alpha-\operatorname{psh}\}
$$

notice that the supremum is non empty by our assumption that $\{\alpha\}$ is pseudoeffective. If $\{\alpha\}$ is big and $\psi$ is $\alpha$-psh and locally bounded in the complement of an analytic $Z \subset X$, one can define the Monge-Ampère measure $\operatorname{MA}_{\alpha}(\psi)$ by

$$
\operatorname{MA}_{\alpha}(\psi):=\mathbb{1}_{X \backslash Z}\left(\alpha+d d^{c} \psi\right)^{n}
$$


as follows from the work of Bedford-Taylor [BT76, BT82]. In particular, if $\{\alpha\}$ is big, there is a well-defined positive measure on $\operatorname{MA}_{\alpha}(\varphi)=\operatorname{MA}_{\alpha}\left(\psi_{\text {min }}\right)$ on $X$; its total mass coincides with $\operatorname{Vol}(\{\alpha\})$, i.e.

$$
\operatorname{Vol}(\{\alpha\})=\int_{X} \operatorname{MA}_{\alpha}(\varphi)
$$

(this follows from the comparison theorem and the fact that Monge-Ampère measures of locally bounded psh functions do not carry mass on analytic sets; see e.g. [BEGZ08]). Next, notice that in general the $\alpha$-psh envelope $\varphi=\psi_{\text {min }}$ corresponds canonically to $\alpha$, so we may associate to $\alpha$ the following subset of $X$ :

$$
D=\{\varphi=0\}
$$

Since $\varphi$ is upper semi-continuous, the set $D$ is compact. Moreover, a simple application of the maximum principle shows that $\alpha \geq 0$ pointwise on $D$ (precisely as in Proposition 3.1 of [Ber07]: at any point $z_{0}$ where $\alpha$ is not semi-positive, we can find complex coordinates and a small $\varepsilon>0$ such that $\varphi(z)-\varepsilon\left|z-z_{0}\right|^{2}$ is subharmonic near $z_{0}$, hence $\left.\varphi\left(z_{0}\right)<0\right)$. In particular, $\mathbb{1}_{D} \alpha$ is a positive $(1,1)$-form on $X$. From Theorem (1.4) we infer

(2.5) Corollary. Assume that $X$ is a Kähler manifold. For any smooth closed form $\alpha$ of type $(1,1)$ in a pseudo-effective class and $\varphi \leqslant 0$ the $\alpha$-psh upper envelope we have

$$
\operatorname{MA}_{\alpha}(\varphi)=\mathbb{1}_{D} \alpha^{n}, \quad D=\{\varphi=0\}
$$

as measures on $X$ (provided the left hand side is interpreted as a suitable weak limit) and

$$
\operatorname{Vol}(\{\alpha\})=\int_{D} \alpha^{n} \geqslant 0
$$

In particular, $\{\alpha\}$ is big if and only if $\int_{D} \alpha^{n}>0$.

Proof. Let $\omega$ be a Kähler metric on $X$. First assume that the class $\{\alpha\}$ is big and let $Z_{0}$ be the singularity set of some strictly positive representative $\alpha+d d^{c} \psi_{0} \geqslant \varepsilon \omega$ with analytic singularities. By Theorem (1.4), $\alpha+d d^{c} \varphi$ is in $L_{\mathrm{loc}}^{\infty}\left(X \backslash Z_{0}\right)$. In particular (see [Dem89]) the Monge-Ampère measure $\left(\alpha+d d^{c} \varphi\right)^{n}$ has a locally bounded density on $X \backslash Z_{0}$ with respect to $\omega^{n}$. Hence, it is enough to prove the identity (2.6) pointwise almost enerywhere on $X$. To this end, one argues essentially as in [Ber07] (where the class was assumed to be integral). First a well-known local argument based on the solution of the Dirichlet problem for $\left(d d^{c}\right)^{n}$ (see e.g. [BT76, BT82], and also Proposition 1.10 in [BB08]) proves that the Monge-Ampère measure $\left(\alpha+d d^{c} \varphi\right)^{n}$ of the envelope $\varphi$ vanishes on the open set $\left(X \backslash Z_{0}\right) \backslash D$ (this only uses the fact that $\alpha$ has continuous potentials and the 
continuity of $\varphi$ on $\left.X \backslash Z_{0}\right)$. Moreover, Theorem (1.4) implies that $\varphi \in C^{1}\left(X \backslash Z_{0}\right)$ and

$$
\frac{\partial^{2} \varphi}{\partial x_{i} \partial x_{j}} \in L_{\mathrm{loc}}^{p}
$$

for any $p \in] 1, \infty[$ and $i, j \in[1,2 n]$. Even if this is slightly weaker than the situation in [Ber07], where it was shown that one can take $p=\infty$, the argument given in [Ber07] still goes through. Indeed, by well-known properties of measurable sets, $D$ has Lebesgue density $\lim _{r \rightarrow 0} \lambda(D \cap B(x, r)) / \lambda(B(x, r)=1$ at almost every point $x \in D$, and since $\varphi=0$ on $D$, we conclude that $\partial \varphi / \partial x_{i}=0$ at those points (if the density is 1 , no open cone of vertex $x$ can be omitted and thus we can approach $x$ from any direction by a sequence $x_{\nu} \rightarrow x$ ). But the first derivative is Hölder continuous on $D \backslash Z_{0}$, hence $\partial \varphi / \partial x_{i}=0$ everywhere on $D \backslash Z_{0}$. By repeating the argument for $\partial \varphi / \partial x_{i}$ which has a derivative in $L^{p}\left(L^{1}\right.$ would even be enough), we conclude from Lebesgue's theorem that $\partial^{2} \varphi / \partial x_{i} \partial x_{j}=0$ a.e. on $D \backslash Z_{0}$, hence $\alpha+d d^{c} \varphi=\alpha$ on $D \backslash E$ where the set $E$ has measure zero with respect to $\omega^{n}$. This proves formula (2.6) in the case of a big class.

Finally, assume that $\{\alpha\}$ is pseudo-effective but not big. For any given positive number $\varepsilon$ we let $\alpha_{\varepsilon}=\alpha+\varepsilon \omega$ and denote by $D_{\varepsilon}$ the corresponding set (2.4). Clearly $\alpha_{\varepsilon}$ represents a big class. Moreover, by the continuity of the volume function up to the boundary of the big cone [Bou02]

$$
\operatorname{Vol}\left(\left\{\alpha_{\varepsilon}\right\}\right) \rightarrow \operatorname{Vol}(\{\alpha\}) \quad(=0)
$$

as $\varepsilon$ tends to zero. Now observe that $D \subset D_{\varepsilon}$ (there are more $(\alpha+\varepsilon \omega)$-psh functions than $\alpha$-psh functions and so $\varphi \leqslant \varphi_{\varepsilon} \leqslant 0$; clearly $\varphi_{\varepsilon}$ increases with $\varepsilon$ and $\varphi=\lim _{\varepsilon \rightarrow 0} \varphi_{\varepsilon}$; compare with Proposition 3.3 in [Ber07]). Therefore

$$
\int_{D} \alpha^{n} \leq \int_{D_{\varepsilon}} \alpha^{n} \leq \int_{D_{\varepsilon}} \alpha_{\varepsilon}^{n},
$$

where we used that $\alpha \leq \alpha_{\varepsilon}$ in the second step. Finally, since by the big case treated above, the right hand side above is precisely $\operatorname{Vol}\left(\left\{\alpha_{\varepsilon}\right\}\right)$, letting $\varepsilon$ tend to zero and using (2.9) proves that $\int_{D} \alpha^{n}=0=\operatorname{Vol}(\{\alpha\})$ [and that $\operatorname{MA}_{\alpha}(\varphi)=0$ if we interpret it as the limit of $\left.\mathrm{MA}_{\alpha_{\varepsilon}}\left(\varphi_{\varepsilon}\right)\right]$. This concludes the proof.

In the case when $\{\alpha\}$ is an integer class, i.e. when it is the first Chern class $c_{1}(L)$ of a holomorphic line bundle $L$ over $X$, the result of the corollary was obtained in [Ber07] under the additional assumption that $X$ be a projective manifold - it was conjectured there that the result was also valid for integral classes over non-projective Kähler manifold.

(2.10) Remark. In particular, the corollary shows that, if $\{\alpha\}$ is big, there is always an $\alpha$-plurisubharmonic function $\varphi$ with minimal singularities such that $\operatorname{MA}_{\alpha}(\varphi)$ has a $L^{\infty}$-density with respect to $\omega^{n}$. This is a very useful fact when dealing with big classes which are not Kähler (see for example [BBGZ09]). 


\section{Application to regularity of a boundary value problem and a variational principle}

In this section we will see how the main theorem may be intepreted as a regularity result for (1) a free boundary value problem for the Monge-Ampère operator and (2) a variational principle. For simplicity we only consider the case of a Kähler class.

\subsection{A free boundary value problem for the Monge-Ampère operator}

Let $(X, \omega)$ be a Kähler manifold. Given a function $f \in \mathrm{C}^{2}(X)$ consider the following free boundary value problem

$$
\left\{\begin{aligned}
\operatorname{MA}_{\omega}(u) & =0 & & \text { on } \Omega, \\
u & =f & & \text { on } \partial \Omega, \\
d u & =d f & &
\end{aligned}\right.
$$

for a pair $(u, \Omega)$, where $u$ is an $\omega$-psh function on $\bar{\Omega}$ which is in $\mathcal{C}^{1}(\bar{\Omega})$ and $\Omega$ is an open set in $X$. We have used the notation $\partial \Omega:=\bar{\Omega} \backslash \Omega$, but no regularity of the boundary is assumed. The reason why the set $\Omega$ is assumed to be part of the solution is that, for a fixed $\Omega$, the equations are overdetermined. Setting $u:=\varphi+f$ and $\Omega:=X \backslash D$ where $\varphi$ is the upper envelope with respect to $\alpha:=d d^{c} f+\omega$, yields a solution. In fact, by Theorem $(1.4) u \in \mathrm{e}^{1,1-\delta}(\bar{\Omega})$ for any $\delta>0$.

\subsection{A variational principle}

Fix a form $\alpha$ in a Kähler class $\{\alpha\}$, possessing continuous potentials. Consider the following energy functional defined on the convex $\operatorname{space} \operatorname{PSH}(X, \alpha) \cap L^{\infty}$ of all $\alpha$-psh functions which are bounded on $X$ :

$$
\mathcal{E}[\psi]:=\frac{1}{n+1} \sum_{j=0}^{n} \int_{X} \psi\left(\alpha+d d^{c} \psi\right)^{j} \wedge \alpha^{n-j}
$$

This functional seems to first have appeared, independently, in the work of Aubin and Mabuchi in Kähler-Einstein geometry (in the case when $\alpha$ is a Kähler form). More geometrically, up to an additive constant, $\mathcal{E}$ can be defined as a primitive of the one form on $\operatorname{PSH}(X, \alpha) \cap L^{\infty}$ defined by the measure valued operator $\psi \mapsto \mathrm{MA}_{\alpha}(\psi)$.

As shown in [BB08] (version 1) the following variational characterization of the envelope $\varphi$ holds:

(3.2.2) Proposition. The functional

$$
\psi \mapsto \mathcal{E}[\psi]-\int_{X} \psi\left(\alpha+d d^{c} \psi\right)^{n}
$$


achieves its minimum value on the space $\operatorname{PSH}(X, \alpha) \cap L^{\infty}$ precisely when $\psi$ is equal to the envelope $\varphi$ (defined with respect to $\alpha$ ). Moreover, the minimum is achieved only at $\varphi$, up to an additive constant.

Hence, the main theorem above can be interpreted as a regularity result for the functions in $\operatorname{PSH}(X, \alpha) \cap L^{\infty}$ minimizing the functional (3.2.1) in the case when $\alpha$ is assumed to have $L_{\text {loc }}^{\infty}$ coefficients. More generally, a similar variational characterization of $\varphi$ can be given the case of a big class $[\alpha]$ [BBGZ09].

\section{Degenerate Monge-Ampère equations and geodesics in the space of Kähler metrics}

Assume that $(X, \omega)$ is a compact Kähler manifold and that $\Sigma$ is a Stein manifold with strictly pseudoconvex boundary, i.e. $\Sigma$ admits a smooth strictly psh non-positive function $\eta_{\Sigma}$ which vanishes precisely on $\partial \Sigma$. The corresponding product manifold will be denoted by $M:=\Sigma \times X$. By taking pull-backs, we identify $\eta_{\Sigma}$ with a function on $M$ and $\omega$ with a semi-positive form on $M$. In this way, we obtain a Kähler form $\omega_{M}:=\omega+d d^{c} \eta_{\Sigma}$ on $M$. Given a function $f$ on $M$ and a point $s$ in $\Sigma$ we use the notation $f_{s}:=f(s, \cdot)$ for the induced function on $X$.

Further, given a closed $(1,1)$ form $\alpha$ on $M$ with bounded coefficients and a continuous function $f$ on $\partial M$, we define the upper envelope:

$$
\varphi_{\alpha, f}:=\sup \left\{\psi: \psi \in \operatorname{PSH}(M, \alpha) \cap C^{0}(M), \psi_{\partial M} \leq f\right\} .
$$

Note that when $\Sigma$ is a point and $f=0$ this definition coincides with the one introduced in section 1. Also, when $F$ is a smooth function on the whole of $M$, the obvious translation $\psi \mapsto \psi^{\prime}=\psi-F$ yields the relation

$$
\varphi_{\beta, f-F}=\varphi_{\alpha, f}-F \quad \text { where } \beta=\alpha+d d^{c} F .
$$

The proof of the following lemma is a straightforward adaptation of the proof of Bedford-Taylor [BT76] in the case when $M$ is a strictly pseudoconvex domain in $\mathbb{C}^{n}$.

(4.3) Lemma. Let $\alpha$ be a closed real $(1,1)$-form on $M$ with bounded coefficients, such that $\alpha_{\mid\{s\} \times X} \geqslant \varepsilon_{0} \omega$ is positive definite for all $s \in \Sigma$. Then the corresponding envelope $\varphi=\varphi_{\alpha, 0}$ vanishes on the boundary of $M$ and is continuous on $M$. Moreover, $M A_{\alpha}(\varphi)$ vanishes in the interior of $M$.

Proof. By (4.2) we have $\varphi_{\alpha, 0}=\varphi_{\beta, 0}+C \eta_{\Sigma}$ where $\beta=\alpha+C d d^{c} \eta_{\Sigma}$ can be taken to be positive definite on $M$ for $C \gg 1$, as is easily seen from the CauchySchwarz inequality and the hypotheses on $\alpha$. Therefore, we can assume without loss of generality that $\alpha$ is positive definite on $M$. Since 0 is a candidate for the supremum defining $\varphi$ it follows immediately that $0 \leq \varphi$ and hence $\varphi_{\partial M}=0$. To see that $\varphi$ is continuous on $\partial M$ (from the inside) take an arbitrary candidate $\psi$ for the sup and observe that

$$
\psi \leq-C \eta_{\Sigma}
$$


for $C \gg 1$, independent of $\psi$. Indeed, since $d d^{c} \psi \geq-\alpha$ there is a large positive constant $C$ such that the function $\psi+C \eta_{\Sigma}$ is strictly plurisubharmonic on $\Sigma \times\{x\}$ for all $x$. Thus the inequality above follows from the maximum principle applied to all slices $\Sigma \times\{x\}$. All in all, taking the sup over all such $\psi$ gives

$$
0 \leq \varphi \leq-C \eta_{\Sigma}
$$

But since $\eta_{\Sigma \mid \partial M}=0$ and $\eta_{\Sigma}$ is continuous it follows that $\varphi\left(x_{i}\right) \rightarrow 0=\varphi(x)$, when $x_{i} \rightarrow x \in \partial M$.

Next, fix a compact subset $K$ in the interior of $M$ and $\varepsilon>0$. Let $M_{\delta}:=\left\{\eta_{\Sigma}<-\delta\right\}$ where $\delta$ is sufficiently small to make sure that $K$ is contained in $M_{4 \delta}$. By the regularization results in [Dem92] or [Dem94], there is a sequence $\varphi_{j}$ in $\operatorname{PSH}\left(M, \alpha-2^{-j} \alpha\right) \cap C^{0}\left(M_{\delta / 2}\right)$ decreasing to the upper semi-continuous regularization $\varphi^{*}$. By replacing $\varphi_{j}$ with $\left(1-2^{-j}\right)^{-1} \varphi_{j}$, we can even assume $\varphi_{j} \in \operatorname{PSH}(M, \alpha) \cap C^{0}\left(M_{\delta / 2}\right)$. Put

$$
\varphi_{j}^{\prime}:=\max \left\{\varphi_{j}-\varepsilon, C \eta_{\Sigma}\right\} \quad \text { on } M_{\delta}, \quad \text { and } \quad \varphi_{j}^{\prime}:=C \eta_{\Sigma} \text { on } M \backslash M_{\delta}
$$

On $\partial M_{\delta}$ we have $C \eta_{\Sigma}=-C \delta$ and we can take $j$ so large that

$$
\varphi_{j}<-C \eta_{\Sigma}+\varepsilon / 2=C \delta+\varepsilon / 2
$$

so we will have $\varphi_{j}-\varepsilon<C \eta_{\Sigma}$ as soon as $2 C \delta \leq \varepsilon / 2$. We simply take $\varepsilon=4 C \delta$. Then $\varphi_{j}^{\prime}$ is a well defined continuous $\alpha$-psh function on $M$, and $\varphi_{j}^{\prime}$ is equal to $\varphi_{j}-\varepsilon$ on $K \subset M_{4 \delta}$ as $C \eta_{\Sigma} \leqslant-4 C \delta \leqslant-\varepsilon \leq \varphi_{j}-\varepsilon$ there. In particular, $\varphi_{j}^{\prime}$ is a candidate for the sup defining $\varphi$, hence $\varphi_{j}^{\prime} \leq \varphi \leq \varphi^{*}$ and so

$$
\varphi^{*} \leq \varphi_{j} \leq \varphi_{j}^{\prime}+\varepsilon \leq \varphi^{*}+\varepsilon
$$

on $K$. This means that $\varphi_{j}$ converges to $\varphi$ uniformly on $K$ and therefore $\varphi$ is continuous on $K$. All in all this shows that $\varphi \in C^{0}(M)$. The last statement of the proposition follows from standard local considerations for envelopes due to Bedford-Taylor [BT76] (see also the exposition made in [Dem89]).

(4.4) Theorem. Let $\alpha$ be a closed real $(1,1)$-form on $M$ with bounded coefficients, such that $\alpha_{\mid\{s\} \times X} \geqslant \varepsilon_{0} \omega$ is positive definite for all $s \in \Sigma$. Consider a continuous function $f$ on $\partial M$ such that $f_{s} \in \operatorname{PSH}\left(X, \alpha_{s}\right)$ for all $s \in \partial \Sigma$. Then the upper envelope $\varphi=\varphi_{\alpha, f}$ is the unique $\alpha$-psh continuous solution of the Dirichlet problem

$$
\varphi=f \quad \text { on } \partial M, \quad\left(d d^{c} u+\alpha\right)^{\operatorname{dim} M}=0 \text { on the interior } M^{\circ} .
$$

Moreover, if $f$ is $C^{1,1}$ on $\partial M$ then, for any $s$ in $\Sigma$, the restriction $\varphi_{s}$ of $\varphi$ on $\{s\} \times X$ has a $d d^{c}$ in $L_{\mathrm{loc}}^{\infty}$. More precisely, we have a uniform bound $\left|d d^{c} \varphi_{s}\right|_{\omega} \leq C$ a.e. on $X$, where $C$ is a constant independent of $s$.

Proof. Without loss of generality, we may assume as in Lemma (4.4) that $\alpha$ is positive definite on $M$. Also, after adding a positive constant to $f$, which only 
has the effect of adding the same constant to $\varphi=\varphi_{\alpha, f}$, we may suppose that $\sup _{\partial M} f>0$ (this will simplify a little bit the arguments below).

Continuity. Let us first prove the continuity statement in the theorem. In the case when $f$ extends to a smooth function $F$ in $\operatorname{PSH}(M,(1-\varepsilon) \alpha)$ the statement follows immediately from (4.2) and Lemma (4.3) since

$$
f-F=0 \text { on } \partial M \text { and } \beta=\alpha+d d^{c} F \geqslant \varepsilon \alpha \geqslant \varepsilon \varepsilon_{0} \omega .
$$

Next, assume that $f$ is smooth on $\partial M$ and that $f_{s} \in \operatorname{PSH}\left(X,(1-\varepsilon) \alpha_{s}\right)$ for all $s \in \partial \Sigma$. If we take a smooth extension $\tilde{f}$ of $f$ to $M$ and $C \gg 1$, we will get

$$
\alpha+d d^{c}\left(\tilde{f}(x, s)+C \eta_{\Sigma}(s)\right) \geqslant(\varepsilon / 2) \alpha
$$

on a sufficiently small neighborhood $V$ of $\partial M$ (again by using Cauchy-Schwarz). Therefore, after enlarging $C$ if necessary, we can define

$$
F(x, s)=\max _{\varepsilon}\left(\tilde{f}(x, s)+C \eta_{\Sigma}(s), 0\right)
$$

with a regularized max function $\max _{\varepsilon}$, in such a way that the maximum is equal to 0 on a neighborhood of $M \backslash V\left(C \gg 1\right.$ being used to ensure that $\widetilde{f}+C \eta_{\Sigma}<0$ on $M \backslash V)$. Then $F$ equals $f$ on $\partial M$ and satisfies

$$
\alpha+d d^{c} F \geqslant(\varepsilon / 2) \alpha \geqslant\left(\varepsilon \varepsilon_{0} / 2\right) \omega
$$

on $M$, and we can argue as previously. Finally, to handle the general case where $f$ is continuous with $f_{s} \in \operatorname{PSH}\left(X, \alpha_{s}\right)$ for every $s \in \Sigma$, we may, by a parametrized version of Richberg's regularization theorem applied to $\left(1-2^{-\nu}\right) f+C 2^{-\nu}$ (see e.g. [Dem91]), write $f$ as a decreasing uniform limit of smooth functions $f_{\nu}$ on $\partial M$ satisfying $f_{\nu, s} \in \operatorname{PSH}\left(X,\left(1-2^{-\nu-1}\right) \alpha_{s}\right)$ for every $s \in \partial \Sigma$. Then $\varphi_{\omega, f}$ is a decreasing uniform limit on $M$ of the continuous functions $\varphi_{\omega, f_{\nu}}$ (as follows easily from the definition of $\varphi_{\omega, f}$ as an upper envelope). Observe also that the uniqueness of a continuous solution of the Dirichlet problem (4.5) results from a standard application of the maximum principle for the Monge-Ampère operator. This proves the general case of the continuity statement.

Smoothness. Next, we turn to the proof of the smoothness statement. Since the proof is a straightforward adaptation of the proof of the main regularity result above we will just briefly indicate the relevant modification. Quite similarly to what we did in section 1 , we consider an $\alpha$-psh function $\psi$ with $\psi \leqslant f$ on $\partial M$, and introduce the fiberwise transform $\Psi_{s}$ of $\psi_{s}$ on each $\{s\} \times X$ which is defined in terms of the exponential map exph $: T_{X} \rightarrow X$, and we put

$$
\Psi(z, s, t)=\Psi_{s}(z, t) .
$$

Then essentially the same calculations as in the previous case show that all properties of $\Psi$ are still valid with the constant $K$ depending on the $C^{1,1}$-norm of the local potentials $u(z, s)$ of $\alpha$, the constant $A$ depending only on $\omega$ and with

$$
\partial \Psi(z, s, t) / \partial(\log t):=\lambda(z, s, t) \rightarrow \nu\left(\psi_{s}\right),
$$


as $t \rightarrow 0^{+}$, where $\nu\left(\psi_{s}\right)$ is the Lelong number of the function $\psi_{s}$ on $X$ at $z$. Moreover, the local vector valued differential $d z$ should be replaced by the differential $d(z, s)=d z+d s$ in the previous formulas. Next, performing a Kiselman-Legendre transform fiberwise we let

$$
\psi_{c, \delta}(z, s):=\left(\psi_{s}\right)_{c, \delta}(z)
$$

Then, using a parametrized version of the estimates of [Dem94] and the properties of $\Psi(z, s, w)$ as in section 1, arguments derived from Kiselman's infimum principle show that

$$
\alpha+d d^{c} \psi_{c, \delta} \geq\left(-A \min (c, \lambda(z, s, \delta))-K \delta^{2}\right) \omega_{M} \geq-\left(A c+K \delta^{2}\right) \omega_{M}
$$

where $\omega_{M}$ is the Kähler form on $M$. In addition to this, we have $\left|\psi_{c, \delta}-f\right| \leqslant K^{\prime} \delta^{2}$ on $\partial M$ by the hypothesis that $f$ is $C^{1,1}$. For a sufficiently large constant $C_{1}$, we infer from this that $\theta=\left(1-C_{1}\left(A c+K \delta^{2}\right)\right) \psi_{c, \delta}$ satisfies $\theta \leqslant f$ on $\partial M$ (here we use the fact that $f>0$ and hence that $\psi_{0} \equiv 0$ is a candidate for the upper envelope). Moreover $\alpha+d d^{c} \theta \geqslant 0$ on $M$ thanks to (4.6) and the positivity of $\alpha$. Therefore $\theta$ is a candidate for the upper envelope and so $\theta \leqslant \varphi=\varphi_{f, \alpha}$. Repeating the arguments of section 1 almost word by word, we obtain for $\left(\rho_{t} \varphi\right)(z, s):=\Phi(z, s, t)$ the analogue of estimate (1.15) which reduces simply to

$$
\liminf _{t \rightarrow 0_{+}} \frac{\rho_{t} \varphi(z, s)-\varphi(z, s)}{t^{2}} \leq C_{2}
$$

as $\psi_{0} \equiv 0$ in the present situation. The final conclusion follows from (1.16) and the related arguments already explained.

In connection to the study of Wess-Zumino-Witten type equations [Don99], [Don02] and geodesics in the space of Kähler metrics [Don99], [Don02], [Che00] it is useful to formulate the result of the previous theorem as an extension problem from $\partial \Sigma$, in the case when $\alpha(z, s)=\omega(z)$ does not depend on $s$. To this end, let $F: \partial \Sigma \rightarrow \operatorname{PSH}(X, \omega)$ be the map defined by $F(s)=f_{s}$. Then the previous theorem gives a continuous "maximal plurisubharmonic" extension $U$ of $F$ to $\Sigma$, where $U(s):=u_{s}$ so that $U: \partial \Sigma \rightarrow \operatorname{PSH}(X, \omega)$.

Let us next specialize to the case when $\Sigma:=A$ is an annulus $R_{1}<|s|<R_{2}$ in $\mathbb{C}$ and the boundary data $f(x, s)$ is invariant under rotations $s \mapsto s e^{i \theta}$. Denote by $f^{0}$ and $f^{1}$ the elements in $\operatorname{PSH}(X, \omega)$ corresponding to the two boundary circles of $A$. Then the previous theorem furnishes a continuous path $f^{t}$ in $\operatorname{PSH}(X, \omega)$, if we put $t=\log |s|$, or rather $t=\log \left(|s| / R_{1}\right) / \log \left(R_{2} / R_{1}\right)$ to be precise. Following [PS08] the corresponding path of semi-positive forms $\omega^{t}:=\omega+d d^{c} f^{t}$ will be called a (generalized) geodesic in $\operatorname{PSH}(X, \omega)$ (compare also with Remark 4.8).

(4.7) Corollary. Assume that the semi-positive closed $(1,1)$ forms $\omega^{0}$ and $\omega^{1}$ belong to the same Kähler class $\{\omega\}$ and have bounded coefficients. Then the 
geodesic $\omega^{t}$ connecting $\omega^{0}$ and $\omega^{1}$ is continuous on $[0,1] \times X$, and there is a constant $C$ such that $\omega^{t} \leq C \omega$ on $X$, i.e. $\omega^{t}$ has uniformly bounded coefficients.

In particular, the previous corollary shows that the space of all semi-positive forms with bounded coefficients, in a given Kähler class, is "geodesically convex".

(4.8) Remark. As shown in the work of Semmes, Mabuchi and Donaldson, the space of Kähler metrics $\mathcal{H}_{\omega}$ in a given Kähler class $\{\omega\}$ admits a natural Riemannian structure defined in the following way (see [Che00] and references therein). First note that the map $u \mapsto \omega+d d^{c} u$ identifies $\mathcal{H}_{\omega}$ with the space of all smooth and strictly $\omega$-psh functions, modulo constants. Now identifying the tangent space of $\mathcal{H}_{\omega}$ at the point $\omega+d d^{c} u \in \mathcal{H}_{\omega}$ with $C^{\infty}(X) / \mathbb{R}$, the squared norm of a tangent vector $v$ at the point $u$ is defined as

$$
\int_{X} v^{2}\left(\omega+d d^{c} u\right)^{n} / n !
$$

Then the potentials $f^{t}$ of any given geodesic $\omega^{t}$ in $\mathcal{H}_{\omega}$ are in fact solutions of the Dirichlet problem (4.5) above, with $\Sigma$ an annulus and $t:=\log |s|$, see [Che00]. However, the existence of a geodesic $u_{t}$ in $\mathcal{H}_{\omega}$ connecting any given points $u_{0}$ and $u_{1}$ is an open and even dubious problem. In the case when $\Sigma$ is a Riemann surface, the boundary data $f$ is smooth with $\alpha_{s}+d d^{c} f_{s}>0$ on $X$ for $s \in \partial \Sigma$ it was shown in [Che00] that the solution $\varphi$ of the Dirichlet problem (4.5) has a total Laplacian which is bounded on $M$. See also [Blo08] for a detailed analysis of the proof in [Che00] and some refinements. On the other hand it is not known whether $\alpha_{s}+d d^{c} \varphi_{s}>0$ for all $s \in \Sigma$, even under the assumption of rotational invariance which appears in the case of geodesics as above. However, see [CT08] for results in this direction. A case similar to the degenerate setting in the previous corollary was also considered very recently in [PS08], building on [Blo08].

(4.9) Remark. Note that the assumption $f \in C^{2}(\partial M)$ is not sufficient to obtain uniform estimates on the total Laplacian on $M$ with respect to $\omega_{M}$ of the envelope $u$ up to the boundary. To see this let $\Sigma$ be the unit-ball in $\mathbb{C}^{2}$ and write $s=\left(s_{1}, s_{2}\right) \in \mathbb{C}^{2}$. Then $f(s):=\left(1+\operatorname{Re} s_{1}\right)^{2-\varepsilon}$ is in $C^{4-2 \varepsilon}(\partial M)$ and $u(x, s):=f(s)$ is the continuous solution of the Dirichlet problem (4.5). However, $u$ is not in $C^{1,1}(M)$ at $(x ;-1,0) \in \partial M$ for any $x \in X$. Note that this exemple is the trivial extension of the exemple in [CNS86] for the real Monge-Ampère equation in the disc.

\section{Regularity of "supercanonical" metrics}

Let $X$ be a compact complex manifold and $\left(L, h_{L, \gamma}\right)$ a holomorphic line bundle over $X$ equipped with a singular hermitian metric $h_{L, \gamma}=e^{-\gamma} h_{L}$ with satisfies $\int e^{-\gamma}<+\infty$ locally on $X$, where $h_{L}$ is a smooth metric on $L$. In fact, we can more generally consider the case where $\left(L, h_{L, \gamma}\right)$ is a "hermitian $\mathbb{R}$-line bundle"; by this we mean that we have chosen a smooth real $d$-closed 
$(1,1)$ form $\alpha_{L}$ on $X$ (whose $d d^{c}$ cohomology class is equal to $c_{1}(L)$ ), and a specific current $T_{L, \gamma}$ representing it, namely $T_{L, \gamma}=\alpha_{L}+d d^{c} \gamma$, such that $\gamma$ is a locally integrable function satisfying $\int e^{-\gamma}<+\infty$. An important special case is obtained by considering a klt (Kawamata log terminal) effective divisor $\Delta$. In this situation $\Delta=\sum c_{j} \Delta_{j}$ with $c_{j} \in \mathbb{R}$, and if $g_{j}$ is a local generator of the ideal sheaf $\mathcal{O}\left(-\Delta_{j}\right)$ identifying it to the trivial invertible sheaf $g_{j} \mathcal{O}$, we take $\gamma=\sum c_{j} \log \left|g_{j}\right|^{2}, T_{L, \gamma}=\sum c_{j}\left[\Delta_{j}\right]$ (current of integration on $\Delta$ ) and $\alpha_{L}$ given by any smooth representative of the same $d d^{c}$-cohomology class; the klt condition precisely means that

$$
\int_{V} e^{-\gamma}=\int_{V} \prod\left|g_{j}\right|^{-2 c_{j}}<+\infty
$$

on a small neighborhood $V$ of any point in the support $|\Delta|=U \Delta_{j}$ (condition (5.1) implies $c_{j}<1$ for every $j$, and this in turn is sufficient to imply $\Delta$ klt if $\Delta$ is a normal crossing divisor; the line bundle $L$ is then the real line bundle $\mathcal{O}(\Delta)$, which makes sens as a genuine line bundle only if $\left.c_{j} \in \mathbb{Z}\right)$. For each klt pair $(X, \Delta)$ such that $K_{X}+\Delta$ is pseudo-effective, H. Tsuji [Ts07a, Ts07b] has introduced a "supercanonical metric" which generalizes the metric introduced by Narasimhan and Simha [NS68] for projective algebraic varieties with ample canonical divisor. We take the opportunity to present here a simpler, more direct and more general approach.

We assume from now on that $K_{X}+L$ is pseudo-effective, i.e. that the class $c_{1}\left(K_{X}\right)+\left\{\alpha_{L}\right\}$ is pseudo-effective, and under this condition, we are going to define a "supercanonical metric" on $K_{X}+L$. Select an arbitrary smooth hermitian metric $\omega$ on $X$. We then find induced hermitian metrics $h_{K_{X}}$ on $K_{X}$ and $h_{K_{X}+L}=h_{K_{X}} h_{L}$ on $K_{X}+L$, whose curvature is the smooth real $(1,1)$-form

$$
\alpha=\Theta_{K_{X}+L, h_{K_{X}+L}}=\Theta_{K_{X}, \omega}+\alpha_{L}
$$

A singular hermitian metric on $K_{X}+L$ is a metric of the form $h_{K_{X}+L, \varphi}=$ $e^{-\varphi} h_{K_{X}+L}$ where $\varphi$ is locally integrable, and by the pseudo-effectivity assumption, we can find quasi-psh functions $\varphi$ such that $\alpha+d d^{c} \varphi \geqslant 0$. The metrics on $L$ and $K_{X}+L$ can now be "subtracted" to give rise to a metric

$$
h_{L, \gamma} h_{K_{X}+L, \varphi}^{-1}=e^{\varphi-\gamma} h_{L} h_{K_{X}+L}^{-1}=e^{\varphi-\gamma} h_{K_{X}}^{-1}=e^{\varphi-\gamma} d V_{\omega}
$$

on $K_{X}^{-1}=\Lambda^{n} T_{X}$, since $h_{K_{X}}^{-1}=d V_{\omega}$ is just the hermitian $(n, n)$ volume form on $X$. Therefore the integral $\int_{X} h_{L, \gamma} h_{K_{X}+L, \varphi}^{-1}$ has an intrinsic meaning, and it makes sense to require that

$$
\int_{X} h_{L, \gamma} h_{K_{X}+L, \varphi}^{-1}=\int_{X} e^{\varphi-\gamma} d V_{\omega} \leqslant 1
$$

in view of the fact that $\varphi$ is locally bounded from above and of the assumption $\int e^{-\gamma}<+\infty$. Observe that condition (5.2) can always be achieved by subtracting 
a constant to $\varphi$. Now, we can generalize Tsuji's supercanonical metrics on klt pairs (cf. [Ts07b]) as follows.

(5.3) Definition. Let $X$ be a compact complex manifold and let $\left(L, h_{L}\right)$ be a hermitian $\mathbb{R}$-line bundle on $X$ associated with a smooth real closed $(1,1)$ form $\alpha_{L}$. Assume that $K_{X}+L$ is pseudo-effective and that $L$ is equipped with a singular hermitian metric $h_{L, \gamma}=e^{-\gamma} h_{L}$ such that $\int e^{-\gamma}<+\infty$ locally on $X$. Take a hermitian metric $\omega$ on $X$ and define $\alpha=\Theta_{K_{X}+L, h_{K_{X}+L}}=\Theta_{K_{X}, \omega}+\alpha_{L}$. Then we define the supercanonical metric $h_{\mathrm{can}}$ of $K_{X}+L$ to be

$$
\begin{aligned}
& h_{K_{X}+L, \text { can }}=\inf _{\varphi} h_{K_{X}+L, \varphi} \text { i.e. } h_{K_{X}+L, \text { can }}=e^{-\varphi_{\text {can }}} h_{K_{X}+L} \text {, where } \\
& \varphi_{\text {can }}(x)=\sup _{\varphi} \varphi(x) \text { for all } \varphi \text { with } \alpha+d d^{c} \varphi \geqslant 0, \quad \int_{X} e^{\varphi-\gamma} d V_{\omega} \leqslant 1 .
\end{aligned}
$$

In particular, this gives a definition of the supercanonical metric on $K_{X}+\Delta$ for every klt pair $(X, \Delta)$ such that $K_{X}+\Delta$ is pseudo-effective, and as an even more special case, a supercanonical metric on $K_{X}$ when $K_{X}$ is pseudo-effective.

In the sequel, we assume that $\gamma$ has analytic singularities, otherwise not much can be said. The mean value inequality then immediately shows that the quasi-psh functions $\varphi$ involved in definition (5.3) are globally uniformly bounded outside of the poles of $\gamma$, and therefore everywhere on $X$, hence the envelopes $\varphi_{\text {can }}=\sup _{\varphi} \varphi$ are indeed well defined and bounded above. As a consequence, we get a "supercanonical" current $T_{\text {can }}=\alpha+d d^{c} \varphi_{\text {can }} \geqslant 0$ and $h_{K_{X}+L \text {, can satisfies }}$

$$
\int_{X} h_{L, \gamma} h_{K_{X}+L, \text { can }}^{-1}=\int_{X} e^{\varphi_{\text {can }}-\gamma} d V_{\omega}<+\infty
$$

It is easy to see that in Definition (5.3) the supremum is a maximum and that $\varphi_{\text {can }}=\left(\varphi_{\text {can }}\right)^{*}$ everywhere, so that taking the upper semicontinuous regularization is not needed. In fact if $x_{0} \in X$ is given and we write

$$
\left(\varphi_{\text {can }}\right)^{*}\left(x_{0}\right)=\limsup _{x \rightarrow x_{0}} \varphi_{\text {can }}(x)=\lim _{\nu \rightarrow+\infty} \varphi_{\text {can }}\left(x_{\nu}\right)=\lim _{\nu \rightarrow+\infty} \varphi_{\nu}\left(x_{\nu}\right)
$$

with suitable sequences $x_{\nu} \rightarrow x_{0}$ and $\left(\varphi_{\nu}\right)$ such that $\int_{X} e^{\varphi_{\nu}-\gamma} d V_{\omega} \leqslant 1$, the wellknown weak compactness properties of quasi-psh functions in $L^{1}$ topology imply the existence of a subsequence of $\left(\varphi_{\nu}\right)$ converging in $L^{1}$ and almost everywhere to a quasi-psh limit $\varphi$. Since $\int_{X} e^{\varphi_{\nu}-\gamma} d V_{\omega} \leqslant 1$ holds true for every $\nu$, Fatou's lemma implies that we have $\int_{X} e^{\varphi-\gamma} d V_{\omega} \leqslant 1$ in the limit. By taking a subsequence, we can assume that $\varphi_{\nu} \rightarrow \varphi$ in $L^{1}(X)$. Then for every $\varepsilon>0$ the mean value $f_{B\left(x_{\nu}, \varepsilon\right)} \varphi_{\nu}$ satisfies

$$
f_{B\left(x_{0}, \varepsilon\right)} \varphi=\lim _{\nu \rightarrow+\infty} f_{B\left(x_{\nu}, \varepsilon\right)} \varphi_{\nu} \geqslant \lim _{\nu \rightarrow+\infty} \varphi_{\nu}\left(x_{\nu}\right)=\left(\varphi_{\text {can }}\right)^{*}\left(x_{0}\right),
$$


hence we get $\varphi\left(x_{0}\right)=\lim _{\varepsilon \rightarrow 0} f_{B\left(x_{0}, \varepsilon\right)} \varphi \geqslant\left(\varphi_{\text {can }}\right)^{*}\left(x_{0}\right) \geqslant \varphi_{\text {can }}\left(x_{0}\right)$, and therefore the sup is a maximum and $\varphi_{\text {can }}=\varphi_{\text {can }}^{*}$.

By elaborating on this argument, we can infer certain regularity properties of the envelope. However, there is no reason why the integral occurring in (5.4) should be equal to 1 when we take the upper envelope. As a consequence, neither the upper envelope nor its regularizations participate to the family of admissible metrics. This is the reason why the estimates that we will be able to obtain are much weaker than in the case of envelopes normalized by a condition $\varphi \leqslant 0$.

(5.5) Theorem. Let $X$ be a compact complex manifold and $\left(L, h_{L}\right)$ a holomorphic $\mathbb{R}$-line bundle such that $K_{X}+L$ is big. Assume that $L$ is equipped with a singular hermitian metric $h_{L, \gamma}=e^{-\gamma} h_{L}$ with analytic singularities such that $\int e^{-\gamma}<+\infty$ (klt condition). Denote by $Z_{0}$ the set of poles of a singular metric $h_{0}=e^{-\psi_{0}} h_{K_{X}+L}$ with analytic singularities on $K_{X}+L$ and by $Z_{\gamma}$ the poles of $\gamma$ (assumed analytic). Then the associated supercanonical metric $h_{\text {can }}$ is continuous on $X \backslash\left(Z_{0} \cup Z_{\gamma}\right)$ and possesses some computable logarithmic modulus of continuity.

Proof. With the notation already introduced, let $h_{K_{X}+L, \varphi}=e^{-\varphi} h_{K_{X}+L}$ be a singular hermitian metric such that its curvature satisfies $\alpha+d d^{c} \varphi \geqslant 0$ and $\int_{X} e^{\varphi-\gamma} d V_{\omega} \leqslant 1$. We apply to $\varphi$ the regularization procedure defined in (1.6). Jensen's inequality implies

$$
e^{\Phi(z, w)} \leqslant \int_{\zeta \in T_{X, z}} e^{\varphi\left(\operatorname{exph}_{z}(w \zeta)\right)} \chi\left(|\zeta|^{2}\right) d V_{\omega}(\zeta)
$$

If we change variables by putting $u=\operatorname{exph}_{z}(w \zeta)$, then in a neighborhood of the diagonal of $X \times X$ we have an inverse map logh : $X \times X \rightarrow T_{X}$ such that $\operatorname{exph}_{z}(\operatorname{logh}(z, u))=u$ and we find for $w$ small enough

$$
\begin{aligned}
\int_{X} e^{\Phi(z, w)-\gamma(z)} d V_{\omega}(z) & \\
& \leqslant \int_{z \in X}\left(\int_{u \in X} e^{\varphi(u)-\gamma(z)} \chi\left(\frac{|\operatorname{logh}(z, u)|^{2}}{|w|^{2}}\right) \frac{1}{|w|^{2 n}} d V_{\omega}(\operatorname{logh}(z, u))\right) d V_{\omega}(z) \\
& =\int_{u \in X} P(u, w) e^{\varphi(u)-\gamma(u)} d V_{\omega}(u)
\end{aligned}
$$

where $P$ is a kernel on $X \times D\left(0, \delta_{0}\right)$ such that

$$
P(u, w)=\int_{z \in X} \frac{1}{|w|^{2 n}} \chi\left(\frac{|\operatorname{logh}(z, u)|^{2}}{|w|^{2}}\right) \frac{e^{\gamma(u)-\gamma(z)} d V_{\omega}(\operatorname{logh}(z, u))}{d V_{\omega}(u)} d V_{\omega}(z) .
$$

Let us first assume that $\gamma$ is smooth (the case where $\gamma$ has logarithmic poles will be considered later). Then a change of variable $\zeta=\frac{1}{w} \operatorname{logh}(z, u)$ shows that $P$ is smooth and we have $P(u, 0)=1$. Since $P(u, w)$ depends only on $|w|$ we infer

$$
P(u, w) \leqslant 1+C_{0}|w|^{2}
$$


for $w$ small. This shows that the integral of $z \mapsto e^{\Phi(z, w)-C_{0}|w|^{2}}$ will be at most equal to 1 , and therefore if we define

$$
\varphi_{c, \delta}(z)=\inf _{t \in] 0, \delta]} \Phi(z, t)+K t^{2}-K \delta^{2}-c \log \frac{t}{\delta}
$$

as in (1.10), the function $\varphi_{c, \delta}(z) \leqslant \Phi(z, \delta)$ will also satisfy

$$
\int_{X} e^{\varphi_{c, \delta}(z)-C_{0} \delta^{2}-\gamma(z)} d V_{\omega} \leqslant 1
$$

Now, thanks to the assumption that $K_{X}+L$ is big, there exists a quasi-psh function $\psi_{0}$ with analytic singularities such that $\alpha+d d^{c} \psi_{0} \geqslant \varepsilon_{0} \omega$. We can assume $\int_{X} e^{\psi_{0}-\gamma} d V_{\omega}=1$ after adjusting $\psi_{0}$ with a suitable constant. Consider a pair of points $x, y \in X$. We take $\varphi$ so that $\varphi(x)=\varphi_{\text {can }}(x)$ (this is possible by the above discussion). We define

$$
\varphi_{\lambda}=\log \left(\lambda e^{\psi_{0}}+(1-\lambda) e^{\varphi}\right)
$$

with a suitable constant $\lambda \in[0,1 / 2]$ which will be fixed later, and obtain in this way regularized functions $\Phi_{\lambda}(z, w)$ and $\varphi_{\lambda, c, \delta}(z)$. This is obviously a compact family and therefore the associated constants $K$ needed in (5.6) are uniform in $\lambda$. Also, as in section 1 , we have

$$
\left.\left.\alpha+d d^{c} \varphi_{\lambda, c, \delta} \geqslant-\left(A c+K \delta^{2}\right) \omega \quad \text { for all } \delta \in\right] 0, \delta_{0}\right]
$$

Finally, we consider the linear combination

$$
\theta=\frac{A c+K \delta^{2}}{\varepsilon_{0}} \psi_{0}+\left(1-\frac{A c+K \delta^{2}}{\varepsilon_{0}}\right)\left(\varphi_{\lambda, c, \delta}-C_{0} \delta^{2}\right)
$$

Clearly, $\int_{X} e^{\varphi_{\lambda}-\gamma} d V_{\omega} \leqslant 1$, and therefore $\theta$ also satisfies $\int_{X} e^{\theta-\gamma} d V_{\omega} \leqslant 1$ by Hölder's inequality. Our linear combination is precisely taken so that $\alpha+d d^{c} \theta \geqslant 0$. Therefore, by definition of $\varphi_{\text {can }}$, we find that

$$
\varphi_{\text {can }} \geqslant \theta=\frac{A c+K \delta^{2}}{\varepsilon_{0}} \psi_{0}+\left(1-\frac{A c+K \delta^{2}}{\varepsilon_{0}}\right)\left(\varphi_{\lambda, c, \delta}-C_{0} \delta^{2}\right) .
$$

Assume $x \in X \backslash Z_{0}$, so that $\varphi_{\lambda}(x)>-\infty$ and $\nu\left(\varphi_{\lambda}, x\right)=0$. In (5.6), the infimum is reached either for $t=\delta$ or for $t$ such that $c=t \frac{d}{d t}\left(\Phi_{\lambda}(z, t)+K t^{2}\right)$. The function $t \mapsto \Phi_{\lambda}(z, t)+K t^{2}$ is convex increasing in $\log t$ and tends to $\varphi_{\lambda}(z)$ as $t \rightarrow 0$. By convexity, this implies

$$
\begin{aligned}
c=t \frac{d}{d t}\left(\Phi_{\lambda}(z, t)+K t^{2}\right) & \leqslant \frac{\left(\Phi_{\lambda}\left(x, \delta_{0}\right)+K \delta_{0}^{2}\right)-\left(\Phi_{\lambda}(z, t)+K t^{2}\right)}{\log \left(\delta_{0} / t\right)} \\
& \leqslant \frac{C_{1}-\varphi_{\lambda}(x)}{\log \left(\delta_{0} / t\right)} \leqslant \frac{C_{1}+\left|\psi_{0}(z)\right|+\log (1 / \lambda)}{\log \left(\delta_{0} / t\right)}
\end{aligned}
$$


hence

$$
\frac{1}{t} \leqslant \max \left(\frac{1}{\delta}, \frac{1}{\delta_{0}} \exp \left(\frac{C_{1}+\left|\psi_{0}(z)\right|+\log (1 / \lambda)}{c}\right)\right)
$$

This shows that $t$ cannot be too small when the infimum is reached. When $t$ is taken equal to the value which achieves the infimum for $z=y$, we find

$$
\varphi_{\lambda, c, \delta}(y)=\Phi_{\lambda}(y, t)+K t^{2}-K \delta^{2}-c \log \frac{t}{\delta} \geqslant \Phi_{\lambda}(y, t)+K t^{2}-K \delta^{2} .
$$

Since $z \mapsto \Phi_{\lambda}(z, t)$ is a convolution of $\varphi_{\lambda}$, we get a bound of the first order derivative

$$
\left|D_{z} \Phi_{\lambda}(z, t)\right| \leqslant\left\|\varphi_{\lambda}\right\|_{L^{1}(X)} \frac{C_{2}}{t} \leqslant \frac{C_{3}}{t},
$$

and with respect to the geodesic distance $d(x, y)$ we infer from this

$$
\Phi_{\lambda}(y, t) \geqslant \Phi_{\lambda}(x, t)-\frac{C_{3}}{t} d(x, y) .
$$

A combination of (5.11), (5.13) and (5.14) yields

$$
\begin{aligned}
\varphi_{\text {can }}(y) & \geqslant \frac{A c+K \delta^{2}}{\varepsilon_{0}} \psi_{0}(y)+\left(1-\frac{A c+K \delta^{2}}{\varepsilon_{0}}\right)\left(\Phi_{\lambda}(x, t)+K t^{2}-K \delta^{2}-\frac{C_{3}}{t} d(x, y)\right) \\
& \geqslant \frac{A c+K \delta^{2}}{\varepsilon_{0}} \psi_{0}(y)+\left(1-\frac{A c+K \delta^{2}}{\varepsilon_{0}}\right)\left(\varphi_{\lambda}(x)-K \delta^{2}-\frac{C_{3}}{t} d(x, y)\right) \\
& \geqslant \log \left(\lambda e^{\psi_{0}(x)}+(1-\lambda) e^{\varphi(x)}\right)-C_{4}\left(\left(c+\delta^{2}\right)\left(\left|\psi_{0}(y)\right|+1\right)+\frac{1}{t} d(x, y)\right), \\
& \geqslant \varphi_{\text {can }}(x)-C_{5}\left(\lambda+\left(c+\delta^{2}\right)\left(\left|\psi_{0}(y)\right|+1\right)+\frac{1}{t} d(x, y)\right),
\end{aligned}
$$

if we use the fact that $\varphi_{\lambda}(x) \leqslant C_{6}, \varphi(x)=\varphi_{\text {can }}(x)$ and $\log (1-\lambda) \geqslant-(2 \log 2) \lambda$ for all $\lambda \in[0,1 / 2]$. By exchanging the roles of $x, y$ and using (5.12), we see that for all $\left.c>0, \delta \in] 0, \delta_{0}\right]$ and $\left.\left.\lambda \in\right] 0,1 / 2\right]$, there is an inequality

$$
\left|\varphi_{\text {can }}(y)-\varphi_{\text {can }}(x)\right| \leqslant C_{5}\left(\lambda+\left(c+\delta^{2}\right)\left(\max \left(\left|\psi_{0}(x)\right|,\left|\psi_{0}(y)\right|\right)+1\right)+\frac{1}{t} d(x, y)\right)
$$

where

$$
\frac{1}{t} \leqslant \max \left(\frac{1}{\delta}, \frac{1}{\delta_{0}} \exp \left(\frac{C_{1}+\max \left(\left|\psi_{0}(x)\right|,\left|\psi_{0}(y)\right|\right)+\log (1 / \lambda)}{c}\right)\right) .
$$

By taking $c, \delta$ and $\lambda$ small, one easily sees that this implies the continuity of $\varphi_{\text {can }}$ on $X \backslash Z_{0}$. More precisely, if we choose

$$
\delta=d(x, y)^{1 / 2}, \lambda=\frac{1}{|\log d(x, y)|}, c=\frac{C_{1}+\max \left(\left|\psi_{0}(x)\right|,\left|\psi_{0}(y)\right|\right)+|\log | \log d(x, y)||}{\log \delta_{0} / d(x, y)^{1 / 2}}
$$


with $d(x, y)<\delta_{0}^{2}<1$, we get $\frac{1}{t} \leqslant d(x, y)^{-1 / 2}$, whence an explicit, but certainly non optimal, modulus of continuity of the form

$$
\left|\varphi_{\text {can }}(y)-\varphi_{\text {can }}(x)\right| \leqslant C_{7}\left(\max \left(\left|\psi_{0}(x)\right|,\left|\psi_{0}(y)\right|\right)+1\right)^{2} \frac{|\log | \log d(x, y)||+1}{|\log d(x, y)|+1} .
$$

When the weight $\gamma$ has analytic singularities, the kernel $P(u, w)$ is no longer smooth and the volume estimate (5.7). In this case, we use a modification $\mu: \widehat{X} \rightarrow X$ in such a way that the singularities of $\gamma \circ \mu$ are divisorial, given by a divisor with normal crossings. If we put

$$
\widehat{L}=\mu^{*} L-K_{\widehat{X} / X}=\mu^{*} L-E
$$

( $E$ the exceptional divisor), then we get an induced singular metric on $\widehat{L}$ which still satisfies the klt condition, and the corresponding supercanonical metric on $K_{\widehat{X}}+\widehat{L}$ is just the pull-back by $\mu$ of the supercanonical metric on $K_{X}+L$. This shows that we may assume from the start that the singularities of $\gamma$ are divisorial and given by a klt divisor $\Delta$. In this case, a solution to the problem is to introduce a complete hermitian metric $\hat{\omega}$ of uniformly bounded curvature on $X \backslash|\Delta|$ by using the Poincaré metric on the punctured disc as a local model transversally to the components of $\Delta$. The Poincaré metric on the punctured unit disc is given by

$$
\frac{|d z|^{2}}{|z|^{2}(\log |z|)^{2}}
$$

and the singularity of $\hat{\omega}$ along the component $\Delta_{j}=\left\{g_{j}(z)=0\right\}$ of $\Delta$ is given by

$$
\hat{\omega}=\sum-d d^{c} \log |\log | g_{j}|| \bmod C^{\infty} .
$$

Since such a metric has bounded geometry and this is all that we need for the calculations of [Dem94] to work, the estimates that we have made here are still valid, especially the crucial lower bound $\alpha+d d^{c} \varphi_{\lambda, c, \delta} \geqslant-\left(A c+K \delta^{2}\right) \hat{\omega}$. In order to compensate this loss of positivity, we need a quasi-psh function $\hat{\psi}_{0}$ such that $\alpha+d d^{c} \hat{\psi}_{0} \geqslant \varepsilon_{0} \hat{\omega}$, but such a lower bound is possible by adding terms of the form $-\varepsilon_{1} \log |\log | g_{j}||$ to our previous quasi-psh function $\psi_{0}$. Now, with respect to the Poincaré metric, a $\delta$-ball of center $z_{0}$ in the punctured disc is contained in the corona

$$
\left|z_{0}\right|^{e^{-\delta}}<|z|<\left|z_{0}\right|^{e^{\delta}}
$$

and it is easy to see from there that the mean value of $|z|^{-2 a}$ on a $\delta$-ball of center $z_{0}$ is multiplied by at most $\left|z_{0}\right|^{-2 a \delta}$. This implies that a function of the form $\hat{\varphi}_{c, \delta}=\varphi_{c, \delta}+C_{9} \delta \sum \log \left|g_{j}\right|$ will actually give rise to an integral $\int_{X} e^{\hat{\varphi}_{c, \delta}-\gamma} d V_{\omega} \leqslant 1$. We see that the term $\delta^{2}$ in (5.15) has to be replaced by a term of the form

$$
\delta \sum \max \left(|\log | g_{j}(x)||,|\log | g_{j}(x)||\right)
$$


This is enough to obtain the continuity of $\varphi_{\text {can }}$ on $X \backslash\left(Z_{0} \cup|\Delta|\right)$, as well as an explicit logarithmic modulus of continuity.

(5.17) Algebraic version. Since the klt condition is open and $K_{X}+L$ is assumed to be big, we can always perturb $L$ a little bit, and after blowing-up $X$, assume that $X$ is projective and that $\left(L, h_{L, \gamma}\right)$ is obtained as a sum of $\mathbb{Q}$-divisors

$$
L=G+\Delta
$$

where $\Delta$ is klt and $G$ is equipped with a smooth metric $h_{G}$ (from which $h_{L, \gamma}$ is inferred, with $\Delta$ as its poles, so that $\left.\Theta_{L, h_{L, \gamma}}=\Theta_{G, L_{G}}+[\Delta]\right)$. Clearly this situation is "dense" in what we have been considering before, just as $\mathbb{Q}$ is dense in $\mathbb{R}$. In this case, it is possible to give a more algebraic definition of the supercanonical metric $\varphi_{\text {can }}$, following the original idea of Narasimhan-Simha [NS68] (see also H. Tsuji [Ts07a]) - the case considered by these authors is the special situation where $G=0, h_{G}=1$ (and moreover $\Delta=0$ and $K_{X}$ ample, for [NS68]). In fact, if $m$ is a large integer which is a multiple of the denominators involved in $G$ and $\Delta$, we can consider sections

$$
\sigma \in H^{0}\left(X, m\left(K_{X}+G+\Delta\right)\right) .
$$

We view them rather as sections of $m\left(K_{X}+G\right)$ with poles along the support $|\Delta|$ of our divisor. Then $(\sigma \wedge \bar{\sigma})^{1 / m} h_{G}$ is a volume form with integrable poles along $|\Delta|$ (this is the klt condition for $\Delta$ ). Therefore one can normalize $\sigma$ by requiring that

$$
\int_{X}(\sigma \wedge \bar{\sigma})^{1 / m} h_{G}=1
$$

Each of these sections defines a singular hermitian metric on $K_{X}+L=K_{X}+G+\Delta$, and we can take the regularized upper envelope

$$
\varphi_{\mathrm{can}}^{\mathrm{alg}}=\left(\sup _{m, \sigma} \frac{1}{m} \log |\sigma|_{h_{K_{X}+L}^{m}}^{2}\right)^{*}
$$

of the weights associated with a smooth metric $h_{K_{X}+L}$. It is clear that $\varphi_{\text {can }}^{\text {alg }} \leqslant \varphi_{\text {can }}$ since the supremum is taken on the smaller set of weights $\varphi=\frac{1}{m} \log |\sigma|_{h_{K_{X}+L}^{m}}^{2}$, and the equalities

$e^{\varphi-\gamma} d V_{\omega}=|\sigma|_{h_{K_{X}+L}^{m}}^{2 / m} e^{-\gamma} d V_{\omega}=(\sigma \wedge \bar{\sigma})^{1 / m} e^{-\gamma} h_{L}=(\sigma \wedge \bar{\sigma})^{1 / m} h_{L, \gamma}=(\sigma \wedge \bar{\sigma})^{1 / m} h_{G}$

imply $\int_{X} e^{\varphi-\gamma} d V_{\omega} \leqslant 1$. We claim that the inequality $\varphi_{\text {can }}^{\text {alg }} \leqslant \varphi_{\text {can }}$ is an equality. The proof is an immediate consequence of the following statement based in turn on the Ohsawa-Takegoshi theorem and the approximation technique of [Dem92].

(5.19) Proposition. With $L=G+\Delta, \omega, \alpha=\Theta_{K_{X}+L, h_{K_{X}+L}}, \gamma$ as above and $K_{X}+L$ assumed to be big, fix a singular hermitian metric $e^{-\varphi} h_{K_{X}+L}$ of curvature $\alpha+d d^{c} \varphi \geqslant 0$, such that $\int_{X} e^{\varphi-\gamma} d V_{\omega} \leqslant 1$. Then $\varphi$ is equal to a regularized limit

$$
\varphi=\left(\limsup _{m \rightarrow+\infty} \frac{1}{m} \log \left|\sigma_{m}\right|_{h_{K_{X}+L}^{m}}^{2}\right)^{*}
$$

for a suitable sequence $\sigma_{m} \in H^{0}\left(X, m\left(K_{X}+G+\Delta\right)\right)$ with $\int_{X}\left(\sigma_{m} \wedge \bar{\sigma}_{m}\right)^{1 / m} h_{G} \leqslant 1$. 
Proof. By our assumption, there exists a quasi-psh function $\psi_{0}$ with analytic singularity set $Z_{0}$ such that

$$
\alpha+d d^{c} \psi_{0} \geqslant \varepsilon_{0} \omega>0
$$

and we can assume $\int_{C} e^{\psi_{0}-\gamma} d V_{\omega}<1$ (the strict inequality will be useful later). For $m \geqslant p \geqslant 1$, this defines a singular metric $\exp \left(-(m-p) \varphi-p \psi_{0}\right) h_{K_{X}+L}^{m}$ on $m\left(K_{X}+L\right)$ with curvature $\geqslant p \varepsilon_{0} \omega$, and therefore a singular metric

$$
h_{L^{\prime}}=\exp \left(-(m-p) \varphi-p \psi_{0}\right) h_{K_{X}+L}^{m} h_{K_{X}}^{-1}
$$

on $L^{\prime}=(m-1) K_{X}+m L$, whose curvature $\Theta_{L^{\prime}, h_{L^{\prime}}} \geqslant\left(p \varepsilon_{0}-C_{0}\right) \omega$ is arbitrary large if $p$ is large enough. Let us fix a finite covering of $X$ by coordinate balls. Pick a point $x_{0}$ and one of the coordinate balls $B$ containing $x_{0}$. By the OhsawaTakegoshi extension theorem applied on the ball $B$, we can find a section $\sigma_{B}$ of $K_{X}+L^{\prime}=m\left(K_{X}+L\right)$ which has norm 1 at $x_{0}$ with respect to the metric $h_{K_{X}+L^{\prime}}$ and $\int_{B}\left|\sigma_{B}\right|_{h_{K_{X}+L^{\prime}}}^{2} d V_{\omega} \leqslant C_{1}$ for some uniform constant $C_{1}$ depending on the finite covering, but independent of $m, p, x_{0}$. Now, we use a cut-off function $\theta(x)$ with $\theta(x)=1$ near $x_{0}$ to truncate $\sigma_{B}$ and solve a $\bar{\partial}$-equation for $(n, 1)$-forms with values in $L$ to get a global section $\sigma$ on $X$ with $\left|\sigma\left(x_{0}\right)\right|_{h_{K_{X}+L^{\prime}}}=1$. For this we need to multiply our metric by a truncated factor $\exp \left(-2 n \theta(x) \log \left|x-x_{0}\right|\right)$ so as to get solutions of $\bar{\partial}$ vanishing at $x_{0}$. However, this perturbs the curvature by bounded terms and we can absorb them again by taking $p$ larger. In this way we obtain

$$
\int_{X}|\sigma|_{h_{K_{X}+L^{\prime}}}^{2} d V_{\omega}=\int_{X}|\sigma|_{h_{K_{X}+L}^{m}}^{2} e^{-(m-p) \varphi-p \psi_{0}} d V_{\omega} \leqslant C_{2} .
$$

Taking $p>1$, the Hölder inequality for congugate exponents $m, \frac{m}{m-1}$ implies

$$
\begin{aligned}
\int_{X}(\sigma \wedge \bar{\sigma})^{\frac{1}{m}} h_{G} & =\int_{X}|\sigma|_{h^{m}{ }_{X}+L}^{2 / m} e^{-\gamma} d V_{\omega} \\
& =\int_{X}\left(|\sigma|_{h_{K_{X}+L}^{m}}^{2} e^{-(m-p) \varphi-p \psi_{0}}\right)^{\frac{1}{m}}\left(e^{\left(1-\frac{p}{m}\right) \varphi+\frac{p}{m} \psi_{0}-\gamma}\right) d V_{\omega} \\
& \leqslant C_{2}^{\frac{1}{m}}\left(\int_{X}\left(e^{\left(1-\frac{p}{m}\right) \varphi+\frac{p}{m} \psi_{0}-\gamma}\right)^{\frac{m}{m-1}} d V_{\omega}\right)^{\frac{m-1}{m}} \\
& \leqslant C_{2}^{\frac{1}{m}}\left(\int_{X}\left(e^{\varphi-\gamma}\right)^{\frac{m-p}{m-1}}\left(e^{\frac{p}{p-1}\left(\psi_{0}-\gamma\right)}\right)^{\frac{p-1}{m-1}} d V_{\omega}\right)^{\frac{m-1}{m}} \\
& \leqslant C_{2}^{\frac{1}{m}}\left(\int_{X} e^{\frac{p}{p-1}\left(\psi_{0}-\gamma\right)} d V_{\omega}\right)^{\frac{p-1}{m}}
\end{aligned}
$$

using the hypothesis $\int_{X} e^{\varphi-\gamma} d V_{\omega} \leqslant 1$ and another application of Hölder's inequality. Since klt is an open condition and $\lim _{p \rightarrow+\infty} \int_{X} e^{\frac{p}{p-1}\left(\psi_{0}-\gamma\right)} d V_{\omega}=$ $\int_{X} e^{\psi_{0}-\gamma} d V_{\omega}<1$, we can take $p$ large enough to ensure that

$$
\int_{X} e^{\frac{p}{p-1}\left(\psi_{0}-\gamma\right)} d V_{\omega} \leqslant C_{3}<1
$$


Therefore, we see that

$$
\int_{X}(\sigma \wedge \bar{\sigma})^{\frac{1}{m}} h_{G} \leqslant C_{2}^{\frac{1}{m}} C_{3}^{\frac{p-1}{m}} \leqslant 1
$$

for $p$ large enough. On the other hand

$$
\left|\sigma\left(x_{0}\right)\right|_{h_{K_{X}+L^{\prime}}}^{2}=\left|\sigma\left(x_{0}\right)\right|_{h_{K_{X}+L}^{m}}^{2} e^{-(m-p) \varphi\left(x_{0}\right)-p \psi_{0}\left(x_{0}\right)}=1,
$$

thus

$$
\frac{1}{m} \log \left|\sigma\left(x_{0}\right)\right|_{h_{K_{X}+L}^{m}}^{2}=\left(1-\frac{p}{m}\right) \varphi\left(x_{0}\right)+\frac{p}{m} \psi_{0}\left(x_{0}\right)
$$

and, as a consequence

$$
\frac{1}{m} \log \left|\sigma\left(x_{0}\right)\right|_{h_{K_{X}+L}^{m}}^{2} \longrightarrow \varphi\left(x_{0}\right)
$$

whenever $m \rightarrow+\infty, \frac{p}{m} \rightarrow 0$, as long as $\psi_{0}\left(x_{0}\right)>-\infty$. In the above argument, we can in fact interpolate in finitely many points $x_{1}, x_{2}, \ldots, x_{q}$ provided that $p \geqslant C_{4} q$. Therefore if we take a suitable dense subset $\left\{x_{q}\right\}$ and a "diagonal" sequence associated with sections $\sigma_{m} \in H^{0}\left(X, m\left(K_{X}+L\right)\right)$ with $m \gg p=p_{m} \gg$ $q=q_{m} \rightarrow+\infty$, we infer that

$$
\left(\limsup _{m \rightarrow+\infty} \frac{1}{m} \log \left|\sigma_{m}(x)\right|_{h_{K_{X}+L}^{m}}^{2}\right)^{*} \geqslant \limsup _{x_{q} \rightarrow x} \varphi\left(x_{q}\right)=\varphi(x)
$$

(the latter equality occurring if $\left\{x_{q}\right\}$ is suitably chosen with respect to $\varphi$ ). In the other direction, (5.20) implies a mean value estimate

$$
\frac{1}{\pi^{n} r^{2 n} / n !} \int_{B(x, r)}|\sigma(z)|_{h_{K_{X}+L}^{m}}^{2} d z \leqslant \frac{C_{5}}{r^{2 n}} \sup _{B(x, r)} e^{(m-p) \varphi+p \psi_{0}}
$$

on every coordinate ball $B(x, r) \subset X$. The function $\left|\sigma_{m}\right|_{h_{K_{X}+L}^{m}}^{2}$ is plurisubharmonic after we correct the non necessarily positively curved smooth metric $h_{K_{X}+L}$ by a factor of the form $\exp \left(C_{6}|z-x|^{2}\right)$, hence the mean value inequality shows that

$$
\frac{1}{m} \log \left|\sigma_{m}(x)\right|_{h_{K_{X}+L}^{m}}^{2} \leqslant \frac{1}{m} \log \frac{C_{5}}{r^{2 n}}+C_{6} r^{2}+\sup _{B(x, r)}\left(1-\frac{p_{m}}{m}\right) \varphi+\frac{p_{m}}{m} \psi_{0} .
$$

By taking in particular $r=1 / m$ and letting $m \rightarrow+\infty, p_{m} / m \rightarrow 0$, we see that the opposite of inequality (5.22) also holds.

(5.23) Remark. We can rephrase our results in slightly different terms. In fact, let us put

$$
\varphi_{m}^{\mathrm{alg}}=\sup _{\sigma} \frac{1}{m} \log |\sigma|_{h_{K_{X}+L}^{m}}^{2}, \quad \sigma \in H^{0}\left(X, m\left(K_{X}+G+\Delta\right)\right),
$$


with normalized sections $\sigma$ such that $\int_{X}(\sigma \wedge \bar{\sigma})^{1 / m} h_{G}=1$. Then $\varphi_{m}^{\text {alg }}$ is quasi-psh (the supremum is taken over a compact set in a finite dimensional vector space) and by passing to the regularized supremum over all $\sigma$ and all $\varphi$ in (5.21) we get

$$
\varphi_{\text {can }} \geqslant \varphi_{m}^{\text {alg }} \geqslant\left(1-\frac{p}{m}\right) \varphi_{\text {can }}(x)+\frac{p}{m} \psi_{0}(x)
$$

As $\varphi_{\text {can }}$ is bounded from above, we find in particular

$$
0 \leqslant \varphi_{\text {can }}-\varphi_{m}^{\text {alg }} \leqslant \frac{C}{m}\left(\left|\psi_{0}(x)\right|+1\right)
$$

This implies that $\left(\varphi_{m}^{\mathrm{alg}}\right)$ converges uniformly to $\varphi_{\text {can }}$ on every compact subset of $X \subset Z_{0}$, and in this way we infer again (in a purely qualitative manner) that $\varphi_{\text {can }}$ is continuous on $X \backslash Z_{0}$. Moreover, we also see that in (5.18) the upper semicontinuous regularization is not needed on $X \backslash Z_{0}$; in case $K_{X}+L$ is ample, it is not needed at all and we have uniform convergence of $\left(\varphi_{m}^{\mathrm{alg}}\right)$ towards $\varphi_{\text {can }}$ on the whole of $X$. Obtaining such a uniform convergence when $K_{X}+L$ is just big looks like a more delicate question, related e.g. to abundance of $K_{X}+L$ on those subvarieties $Y$ where the restriction $\left(K_{X}+L\right)_{\mid Y}$ would be e.g. nef but not big.

(5.24) Generalization. In the general case where $L$ is a $\mathbb{R}$-line bundle and $K_{X}+L$ is merely pseudo-effective, a similar algebraic approximation can be obtained. We take instead sections

$$
\sigma \in H^{0}\left(X, m K_{X}+\lfloor m G\rfloor+\lfloor m \Delta\rfloor+p_{m} A\right)
$$

where $\left(A, h_{A}\right)$ is a positive line bundle, $\Theta_{A, h_{A}} \geqslant \varepsilon_{0} \omega$, and replace the definition of $\varphi_{\text {can }}^{\text {alg by }}$

$$
\begin{aligned}
& \varphi_{\mathrm{can}}^{\mathrm{alg}}=\left(\limsup _{m \rightarrow+\infty} \sup _{\sigma} \frac{1}{m} \log |\sigma|_{h_{m K_{X}+\lfloor m G\rfloor+p_{m} A}^{2}}\right)^{*}, \\
& \int_{X}(\sigma \wedge \bar{\sigma})^{\frac{2}{m}} h_{\lfloor m G\rfloor+p_{m} A}^{\frac{1}{m}} \leqslant 1,
\end{aligned}
$$

where $m \gg p_{m} \gg 1$ and $h_{\lfloor m G\rfloor}^{1 / m}$ is chosen to converge uniformly to $h_{G}$.

We then find again $\varphi_{\text {can }}=\varphi_{\text {can }}^{\text {alg }}$, with an almost identical proof - though we no longer have a sup in the envelope, but just a lim sup. The analogue of Proposition (5.19) also holds true in this context, with an appropriate sequence of sections $\sigma_{m} \in H^{0}\left(X, m K_{X}+\lfloor m G\rfloor+\lfloor m \Delta\rfloor+p_{m} A\right)$.

(5.27) Remark. The envelopes considered in section 1 are envelopes constrained by an $L^{\infty}$ condition, while the present ones are constrained by an $L^{1}$ condition. It is possible to interpolate and to consider envelopes constrained by an $L^{p}$ condition. 
More precisely, assuming that $\frac{1}{p} K_{X}+L$ is pseudo-effective, we look at metrics $e^{-\varphi} h_{\frac{1}{p} K_{X}+L}$ and normalize them with the $L^{p}$ condition

$$
\int_{X} e^{p \varphi-\gamma} d V_{\omega} \leqslant 1
$$

This is actually an $L^{1}$ condition for the induced metric on $p L$, and therefore we can just apply the above after replacing $L$ by $p L$. If we assume moreover that $L$ is pseudo-effective, it is clear that the $L^{p}$ condition converges to the $L^{\infty}$ condition $\varphi \leqslant 0$, if we normalize $\gamma$ by requiring that $\int_{X} e^{-\gamma} d V_{\omega}=1$.

(5.28) Remark. It would be nice to have a better understanding of the supercanonical metrics. In case $X$ is a curve, this should be easier. In fact $X$ then has a hermitian metric $\omega$ with constant curvature, which we normalize by requiring that $\int_{X} \omega=1$, and we can also suppose $\int_{X} e^{-\gamma} \omega=1$. The class $\lambda=c_{1}\left(K_{X}+L\right) \geqslant 0$ is a number and we take $\alpha=\lambda \omega$. Our envelope is $\varphi_{\text {can }}=\sup \varphi$ where $\lambda \omega+d d^{c} \varphi \geqslant 0$ and $\int_{X} e^{\varphi-\gamma} \omega \leqslant 1$. If $\lambda=0$ then $\varphi$ must be constant and clearly $\varphi_{\text {can }}=0$. Otherwise, if $G(z, a)$ denotes the Green function such that $\int_{X} G(z, a) \omega(z)=0$ and $d d^{c} G(z, a)=\delta_{a}-\omega(z)$, we find

$$
\varphi_{\text {can }}(z) \geqslant \sup _{a \in X}\left(\lambda G(z, a)-\log \int_{z \in X} e^{\lambda G(z, a)-\gamma(z)} \omega(z)\right)
$$

by taking already the envelope over $\varphi(z)=\lambda G(z, a)$ - Const. It is natural to ask whether this is always an equality, i.e. whether the extremal functions are always given by one of the Green functions, especially when $\gamma=0$.

\section{References}

[BT76] Bedford, E. and Taylor, B.A.: The Dirichlet problem for the complex Monge-Ampère equation; Invent. Math. 37 (1976) 1-44.

[BT82] Bedford, E. and Taylor, B.A.: A new capacity for plurisubharmonic functions; Acta Math. 149 (1982) 1-41.

[Ber07] Berman, R.: Bergman kernels and equilibrium measures for line bundles over projective manifolds; arXiv:0710.4375, to appear in the American Journal of Mathematics.

[BB08] Berman, R., Boucksom, S.: Growth of balls of holomorphic sections and energy at equilibrium; arXiv:0803.1950.

[BBGZ09] Berman, R., Boucksom, S., Guedj, V., Zeriahi, A.: A variational approach to solving complex Monge-Ampère equations; in preparation.

[Blo09] Błocki, Z: On geodesics in the space of Kähler metrics; Preprint 2009 available at http://gamma.im.uj.edu.pl/ blocki/publ/.

[Bou02] Boucksom, S.: On the volume of a line bundle; Internat. J. Math 13 (2002), 10431063.

[BEGZ08] Boucksom, S., Eyssidieux, P., Guedj, V., Zeriahi, A.: Monge-Ampère equations in big cohomology classes; arXiv:0812.3674.

[CNS86] Caffarelli, L., Nirenberg, L., Spruck, J.: The Dirichlet problem for the degenerate Monge-Ampère equation; Rev. Mat. Iberoamericana 2 (1986), 19-27. 
[Che00] Chen, X.:The space of Kähler metrics; J. Differential Geom. 56 (2000), 189-234.

[CT08] Chen, X. X., Tian, G.: Geometry of Kähler metrics and foliations by holomorphic discs; Publ. Math. Inst. Hautes Études Sci. 107 (2008), 1-107.

[Dem89] Demailly, J.-P.: Potential Theory in Several Complex Variables; Manuscript available at www-fourier.ujf-grenoble.fr/ demailly/books.html, [D3].

[Dem91] Demailly, J.-P.: Complex analytic and algebraic geometry; manuscript Institut Fourier, first edition 1991, available online at http://www-fourier.ujf-grenoble.fr/ demailly/books.html.

[Dem92] Demailly, J.-P.: Regularization of closed positive currents and Intersection Theory; J. Alg. Geom. 1 (1992), 361-409.

[Dem93] Demailly, J.-P.: Monge-Ampère operators, Lelong numbers and intersection theory; Complex Analysis and Geometry, Univ. Series in Math., edited by V. Ancona and A. Silva, Plenum Press, New-York, 1993.

[Dem94] Demailly, J.-P.: Regularization of closed positive currents of type $(1,1)$ by the flow of a Chern connection; Actes du Colloque en l'honneur de P. Dolbeault (Juin 1992), édité par H. Skoda et J.-M. Trépreau, Aspects of Mathematics, Vol. E 26, Vieweg, 1994, 105-126.

[Don99] Donaldson, S.K.: Symmetric spaces, Kähler geometry and Hamiltonian dynamics; Northern California Symplectic Geometry Seminar, 13-33, Amer. Math. Soc. Transl. Ser. 2, 196, Amer. Math. Soc., Providence, RI, 1999.

[Don02] Donaldson, S.K.: Holomorphic discs and the complex Monge-Ampère equation; J. Symplectic Geom. 1 (2002), 171-196.

[Kis78] Kiselman, C. O.: The partial Legendre transformation for plurisubharmonic functions; Inventiones Math. 49 (1978) 137-148.

[Kis94] Kiselman, C. O.: Attenuating the singularities of plurisubharmonic functions; Ann. Polonici Mathematici 60 (1994) 173-197.

[NS68] Narasimhan, M.S., Simha, R.R.: Manifolds with ample canonical class; Inventiones Math. 5 (1968) 120-128.

[OhT87] Ohsawa, T., Takegoshi, K.: On the extension of $L^{2}$ holomorphic functions; Math. Zeitschrift 195 (1987) 197-204.

[PS08] Phong, D.H., Sturm. J: The Dirichlet problem for degenerate complex MongeAmpère equations; arXiv:0904.1898.

[Rud66] Rudin, W.: Real and complex analysis; McGraw-Hill, 1966, third edition 1987.

[Ts07a] Tsuji, H.: Canonical singular hermitian metrics on relative canonical bundles; arXiv:0704.0566.

[Ts07b] Tsuji, H.: Canonical volume forms on compact Kähler manifolds; arXiv:0707.0111.

(version of May 5, 2009, printed on October 17, 2018) 